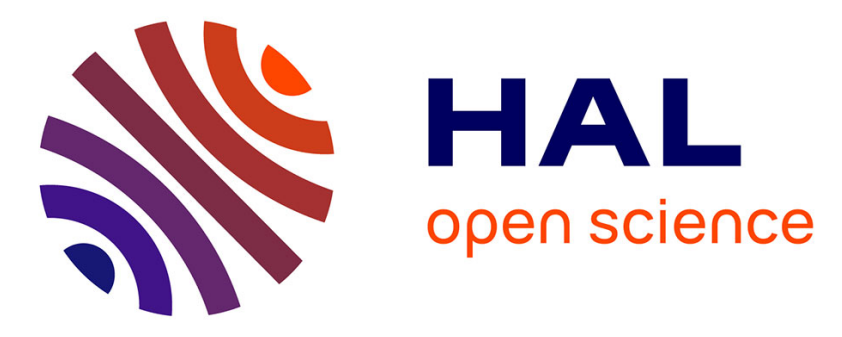

\title{
Premières observations sur le gisement gravettien à statuettes féminines d'Amiens-Renancourt 1 (Somme)
}

Clément Paris, Émeline Deneuve, Jean-Pierre Fagnart, Paule Coudret, Pierre Antoine, Caroline Peschaux, Jessica Lacarrière, Sylvie Coutard, Olivier Moine, Gilles Guérin

\section{To cite this version:}

Clément Paris, Émeline Deneuve, Jean-Pierre Fagnart, Paule Coudret, Pierre Antoine, et al.. Premières observations sur le gisement gravettien à statuettes féminines d'Amiens-Renancourt 1 (Somme). Bulletin de la Société préhistorique française, 2017, 114 (3), pp.423-444. 10.3406/bspf.2017.14801 . hal-02056808

\section{HAL Id: hal-02056808 https://hal.science/hal-02056808}

Submitted on 4 Mar 2019

HAL is a multi-disciplinary open access archive for the deposit and dissemination of scientific research documents, whether they are published or not. The documents may come from teaching and research institutions in France or abroad, or from public or private research centers.
L'archive ouverte pluridisciplinaire HAL, est destinée au dépôt et à la diffusion de documents scientifiques de niveau recherche, publiés ou non, émanant des établissements d'enseignement et de recherche français ou étrangers, des laboratoires publics ou privés. 


\title{
Premières observations sur le gisement gravettien à statuettes féminines d'Amiens-Renancourt 1 (Somme)
}

\author{
Clément Paris, Émeline Deneuve, Jean-Pierre Fagnart, Paule Coudret, Pierre Antoine, \\ Caroline Peschaux, Jessica Lacarrière, Sylvie Coutard, Olivier Moine et Gilles Guérin
}

Résumé : Le quartier de Renancourt, situé à l'ouest de la ville d'Amiens, est connu dans la littérature archéologique depuis le début du $\mathrm{XX}^{\mathrm{e}}$ siècle par les travaux de V. Commont menés dans « l'ancienne briqueterie Devalois ». Jusqu'à une date récente, ce gisement de plein air est resté l'un des rares témoignages du Paléolithique supérieur ancien pour l'ensemble de la région loessique du Nord de la France. À partir des années 1990 et plus particulièrement dès 2010, la découverte de plusieurs gisements dans le cadre de l'archéologie préventive a permis de mieux documenter et d'enrichir notre connaissance de cette période. En 2011, une nouvelle concentration de vestiges, découverte à proximité immédiate des premières fouilles de V. Commont a été mise au jour lors d'un diagnostic archéologique lié à un projet d'aménagement. Ce gisement, désormais appelé Amiens-Renancourt 1, fait l'objet depuis 2014 de campagnes de fouilles programmées annuelles.

L'occupation archéologique, située à $4 \mathrm{~m}$ de profondeur, est incluse dans un gley de toundra. L'ensemble de la séquence loessique, d'une puissance d'environ $8 \mathrm{~m}$, correspond en grande partie au Pléniglaciaire supérieur weichselien. Les premières observations taphonomiques témoignent d'une nappe de vestiges rapidement recouverte par la sédimentation loessique. Six datations ${ }^{14} \mathrm{C}$ sont maintenant disponibles et placent l'occupation entre 22000 et $23000 \mathrm{BP}$, soit aux alentours de 27000 ans cal. BP.

La surface fouillée couvre actuellement $41 \mathrm{~m}^{2}$. Le mobilier lithique et osseux, particulièrement riche, s'organise en différentes concentrations dont certaines peuvent atteindre plusieurs centaines de vestiges au mètre carré. Les restes osseux sont assez bien conservés et parmi le spectre faunique, le cheval apparaît comme l'espèce dominante. L'industrie lithique est réalisée dans un silex d'excellente qualité, disponible aux abords immédiats du site. Elle est caractérisée par une production de grandes lames, dépassant parfois les $20 \mathrm{~cm}$, obtenues au percuteur organique tendre, pour la fabrication de l'outillage commun mais également de quelques armatures (pointes de la Gravette). Une production lamellaire autonome est dévolue à la fabrication des armatures composées de fragments de pièces à dos à retouche abrupte et de quelques microgravettes.

À ces vestiges s'ajoute la découverte exceptionnelle de plusieurs statuettes féminines en craie et d'éléments de parure qui font l'objet d'une première description dans le cadre de cet article. Les statuettes, entières ou fragmentées, sont de dimensions variées. Elles partagent les mêmes particularités stylistiques, à savoir des caractères féminins exagérément prononcés avec une poitrine souvent opulente et des fesses parfois projetées vers l'arrière. Réalisées dans une craie assez tendre, l'état de conservation souvent fragmentaire de ces représentations humaines autorise cependant une bonne lecture des stigmates de fabrication. Compte tenu du matériau utilisé, la reconstitution de la chaîne opératoire de fabrication de ces statuettes pourra aisément inclure la réalisation de tests expérimentaux et apporter des hypothèses d'interprétation quant à la fragmentation de ces objets mobiliers.

Le site a également livré des parures sous forme de rondelles en craie percées et parfois rainurées sur leur pourtour. Plusieurs moules internes silicifiés de Turritelles peuvent également être rapprochés de cette catégorie des parures. Ils proviennent probablement des affleurements lutétiens de la vallée de l'Aisne ou de la Marne, situés à environ $100 \mathrm{~km}$ au sud-est du gisement. La poursuite des recherches sur un gisement bien conservé comme celui d'Amiens-Renancourt 1 contribuera à une meilleure connaissance du peuplement et du modèle socio-économique des groupes de chasseurs qui ont occupé le Nord de la France avant le second maximum de froid du Weichselien. Préalablement rapportée au Gravettien final, l'attribution chronoculturelle s'oriente aujourd'hui davantage vers un faciès du Gravettien récent ou récent-final, qui reste à mieux définir.

Mots clés : Paléolithique supérieur ancien, Nord de la France, Gravettien récent-final, statuettes féminines, parure, lœss, Pléniglaciaire supérieur weichselien.

Abstract : The Renancourt district, located to the west of the town of Amiens, has been known in archaeological literature since the beginning of the 20th century through work carried out by V. Commont in the 'ancienne briqueterie 
Devalois'0. Until recently, this open-air site was one of the rare early Upper Palaeolithic records for the whole loess region in the north of France. From the 1990s onwards, and particularly from 2010, the discovery of several sites as part of rescue archaeological operations enhanced the record and improved our knowledge of this period. In 2011, a new concentration of remains, discovered right beside the first excavations undertaken by V. Commont, was brought to light during archaeological assessments linked to a development project. Annual programmed excavations have been conducted since 2014 at this site, now called Amiens-Renancourt 1.

The archaeological occupation, located at a depth of $4 \mathrm{~m}$, is in a tundra gley. The loess sequence is about $8 \mathrm{~m}$ thick and corresponds mainly to the Upper Weichselian Pleniglacial. The first taphonomic observations point to a layer of remains covered rapidly by loess sedimentation. Six radiocarbon dates are now available and place the occupation between 22000 and 23000 BP, i.e. around 27000 cal. BP.

The excavated surface currently extends over $41 \mathrm{~m}^{2}$. The abundant lithic and bone objects are organized into different concentrations, some of which attain several hundred remains per square metre. The bone remains are relatively well conserved and the dominant species in the faunal spectrum is horse. The lithic industry is in high-quality flint, available immediately beside the site. It is characterized by the production of large blades, sometimes over $20 \mathrm{~cm}$ long, obtained with a soft organic hammer, for manufacturing common tools but also several microliths (Gravette points). Separate bladelet production is used for the production of microliths, made up of backed bladelets with abrupt retouch, and several microgravettes.

Alongside these remains, several exceptional female statuettes in chalk were discovered with some ornamental elements. A first description of these objects is presented in this article. The whole or fragmented statuettes are of varied dimensions. They show the same stylistic characteristics, that is, overstated feminine attributes, often with full breasts and sometimes buttocks projected towards the rear. They are in fairly tender chalk and are often fragmented but are nonetheless conducive to the legible interpretation of manufacturing marks. Considering the material used, the reconstruction of the chaine opératoire of these figurines could easily include experimental tests and provide interpretative hypotheses for the fragmentation of these objects. Two types of personal ornaments were also discovered on the site: perforated roundels in chalk, some of which are grooved around the edge, and internal silicified Turritella fossils which probably come from Lutetian outcrops from the Aisne or Marne valley, located about $100 \mathrm{~km}$ south-east of the site.

Continued research at the 'high resolution' site of Amiens-Renancourt 1 will contribute to enhancing our knowledge of the settlement and socio-economic model of groups of hunters in the north of France before the second Weichselian glacial maximum. Previous chrono-cultural attributions pointed to a Final Gravettian age for this site, but current work indicates a Late-Final Gravettian age, which now requires further clarification.

Keywords : Early Upper Palaeolithic, Northern France, Late-Final Gravettian, female statuettes, ornaments, lœss, weichselian Upper Pleniglacial.

$\mathrm{J}$ USQU'AU début du XXI ${ }^{e}$ siècle, les études sur le Paléolithique supérieur ancien du Nord de la France (région Hauts-de-France) se sont heurtées à une déficience ou une insuffisance des données. En effet, jusqu'à une date récente, peu de sites pouvaient être rattachés à cette période et la documentation recueillie, souvent réduite d'un point de vue quantitatif, ne bénéficiait pas d'un contexte particulièrement explicite. L'apport scientifique de ces ensembles issus de ramassages de surface ou de fouilles anciennes restait donc limité (Fagnart et al., 2013).

Ces dernières années, de nouveaux gisements inclus dans d'importantes séquences lœssiques ont été découverts dans le cadre de l'archéologie préventive (Goval et Hérisson, 2012; Paris et al., 2013). Leur préservation dans des contextes particulièrement favorables a permis de préciser le cadre chronostratigraphique des occupations archéologiques, étayé par un corpus de datations absolues et de données paléoenvironnementales. Par ailleurs, les vestiges recueillis et leur organisation intra-site laissent envisager à terme une approche palethnologique des occupations et une meilleure compréhension du mode de peuplement régional.

La découverte en 2011 du gisement d'Amiens-Renancourt 1, lors d'un diagnostic archéologique réalisé par l'INRAP, entre dans le cadre de cette nouvelle dynamique (Paris et al., 2013). N'étant pas menacé dans l'immédiat par le projet d'aménagement de la ZAC de Renancourt, le gisement a fait l'objet d'un sondage complémentaire en 2013 puis d'un programme annuel de fouilles à partir de 2014.

Les premières données contextuelles recueillies lors de ces différentes interventions (stratigraphie, datations, taphonomie) sont présentées ici, ainsi que la découverte exceptionnelle de statuettes et d'éléments de parure en craie. Toutefois, ces données revêtent aujourd'hui un caractère préliminaire et seront complétées par des études ultérieures.

\section{LOCALISATION ET CONTEXTE GÉOLOGIQUE DU GISEMENT}

\footnotetext{
Situé dans le quartier Renancourt à l'ouest d'Amiens, le gisement est localisé dans la partie inférieure d'un promontoire limoneux délimité au sud-ouest par la vallée de la Selle et au nord par un vallon sec très encaissé, la "vallée de Grâce ». L'ensemble domine la vallée de la Selle à quelques centaines de mètres de sa confluence avec la Somme. Le site d'Amiens-Renancourt 1 se situe plus précisément à la base du versant septentrional de ce promontoire orienté vers la «vallée de Grâce » (fig. 1).
} 


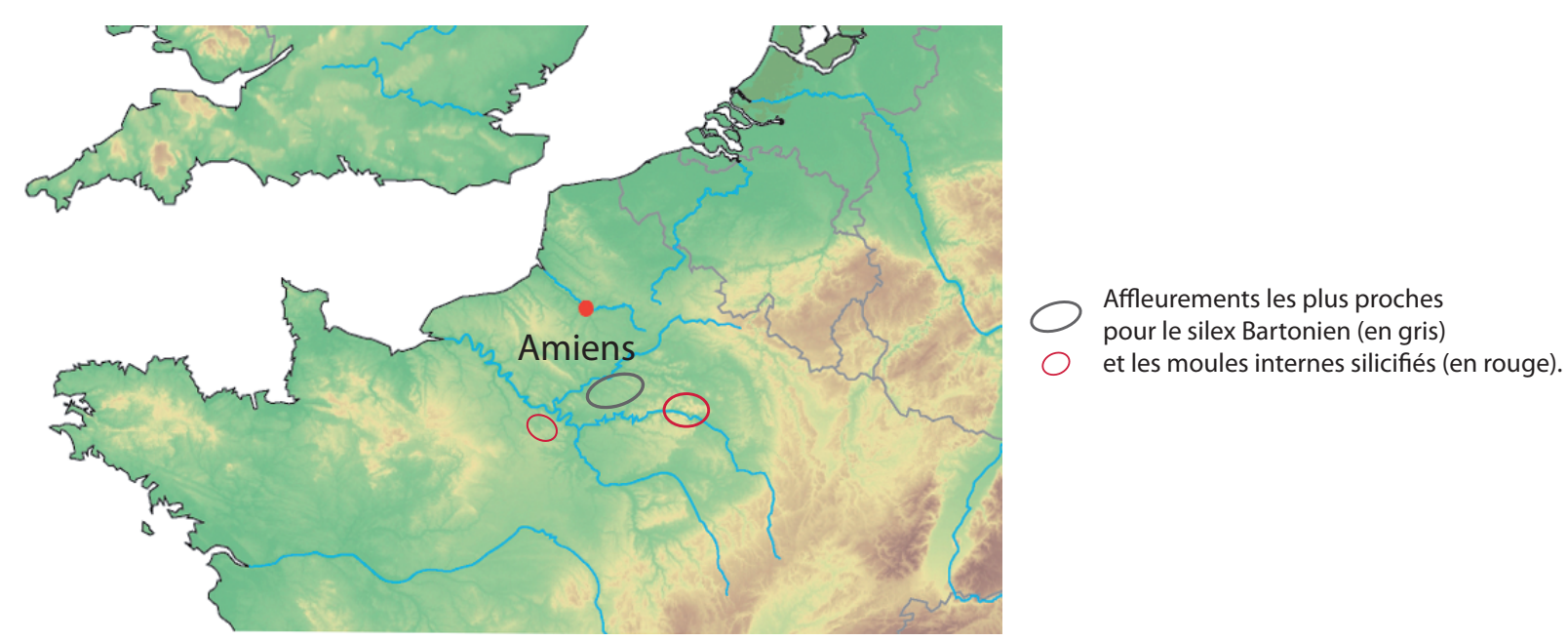

Légende

Fond de vallée

Talus ou briqueterie

Emprises

diagnostiquées

depuis 1996

Délimitation des gisements du Paléolithique supérieur ancien
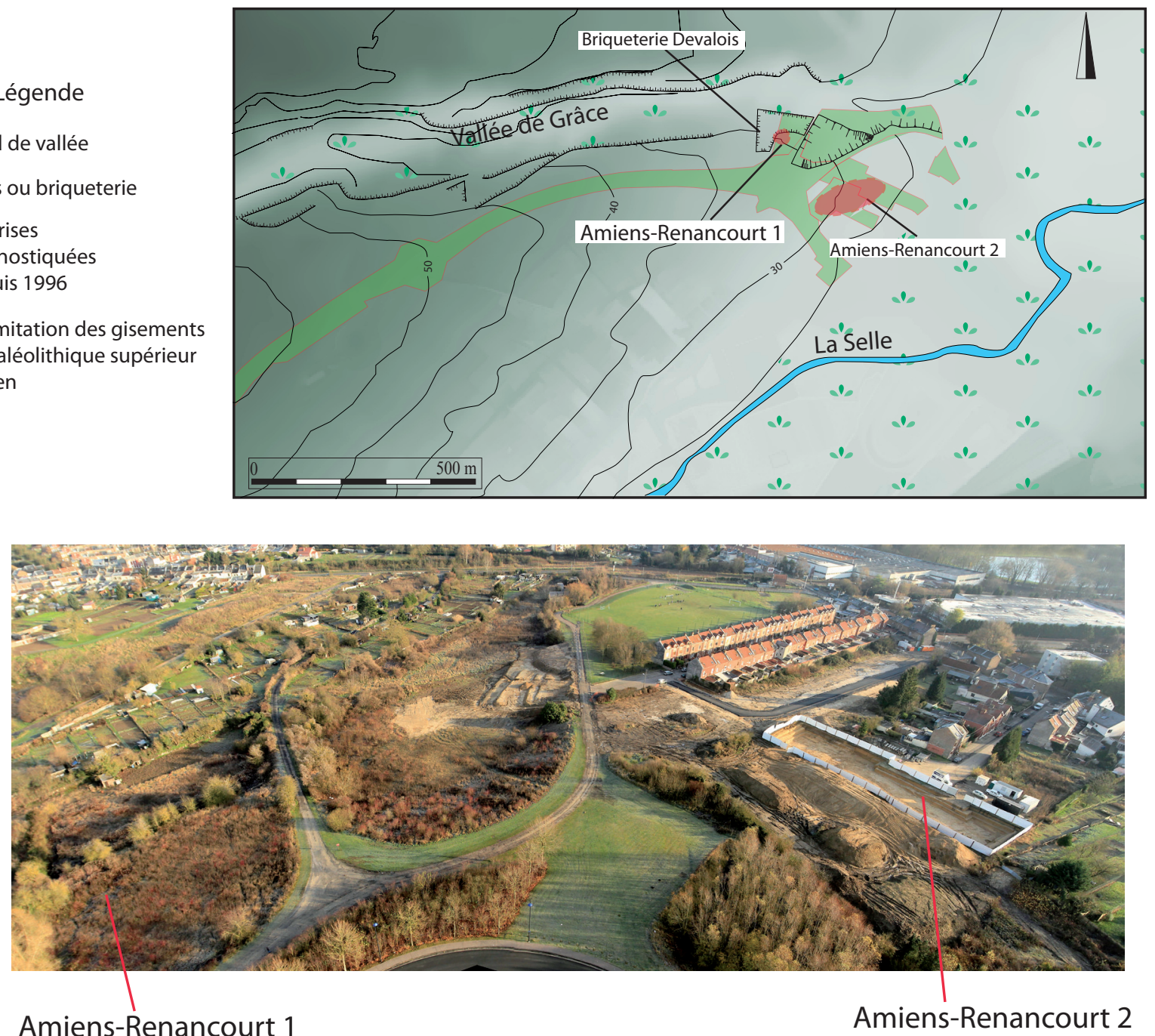

Fig. 1 - Localisation géographique des gisements d'Amiens-Renancourt 1 et 2 et photographie aérienne des deux secteurs fouillés (C) J. Refuveille, Balloïde).

Fig. 1 - Geographic location of the sites of Amiens-Renancourt 1 and 2 and aerial photo of the two excavated sectors (C) J. Refuveille, Balloïde). 
Depuis 2007, de nombreux sondages profonds ont largement contribué à enrichir les connaissances géomorphologiques du secteur. Elles ont été intégrées dans un système d'information géographique (SIG) qui permet de visualiser et de mieux comprendre l'évolution du versant au Pléistocène (Font et al., 2016). Ainsi, la cartographie du toit du substrat crayeux montre que le gisement d'Amiens-Renancourt 1 est implanté en bas de versant, au pied d'un talus crayeux qui forme à cet endroit un éperon descendant vers la vallée de Grâce (fig. 2). Cette avancée forme une protection naturelle en demicercle qui est favorable à la conservation des occupations archéologiques. En favorisant ainsi le développement végétal et le maintien de l'humidité locale, elle a donc favorisé l'accumulation des lœss (près de huit mètres dans ce secteur) et limité leur reprise sédimentaire par des phénomènes de déflation. Le talus crayeux joue également un rôle attractif dans le choix de l'implantation du campement par les hommes du Paléolithique. Il assure une protection contre les vents et offre une potentielle zone d'acquisition de matières premières siliceuses et crayeuses. En effet, cet affleurement, tout comme les versants abrupts de la vallée de Grâce à proximité, sont riches en silex de bonne qualité provenant des étages crétacés du Turonien supérieur-Coniacien basal et du Coniacien. Les alluvions et dépôts de bas de versant environnants abondent également de blocs de silex en position secondaire.

\section{HISTORIQUE DES RECHERCHES}

T e quartier de Renancourt est connu pour ses extracLtions de « terre à briques » à la fin du XIX ${ }^{\mathrm{e}}$ et au début du XX $X^{\mathrm{e}}$ siècle. Dès 1901, L. Delambre et M. Delaire récoltent une série de lames et d'outils en silex associés à des ossements de chevaux dans les labours destinés à mettre en culture le fond d'une de ces anciennes exploitations dénommée « briqueterie Devalois ». Suite à ces découvertes, V. Commont effectue en octobre 1910 une fouille sur une surface de $150 \mathrm{~m}^{2}$. Un niveau bien individualisé est localisé à $4 \mathrm{~m}$ de la surface du sol d'origine (fig. 3). L'industrie est incluse dans un petit cailloutis de

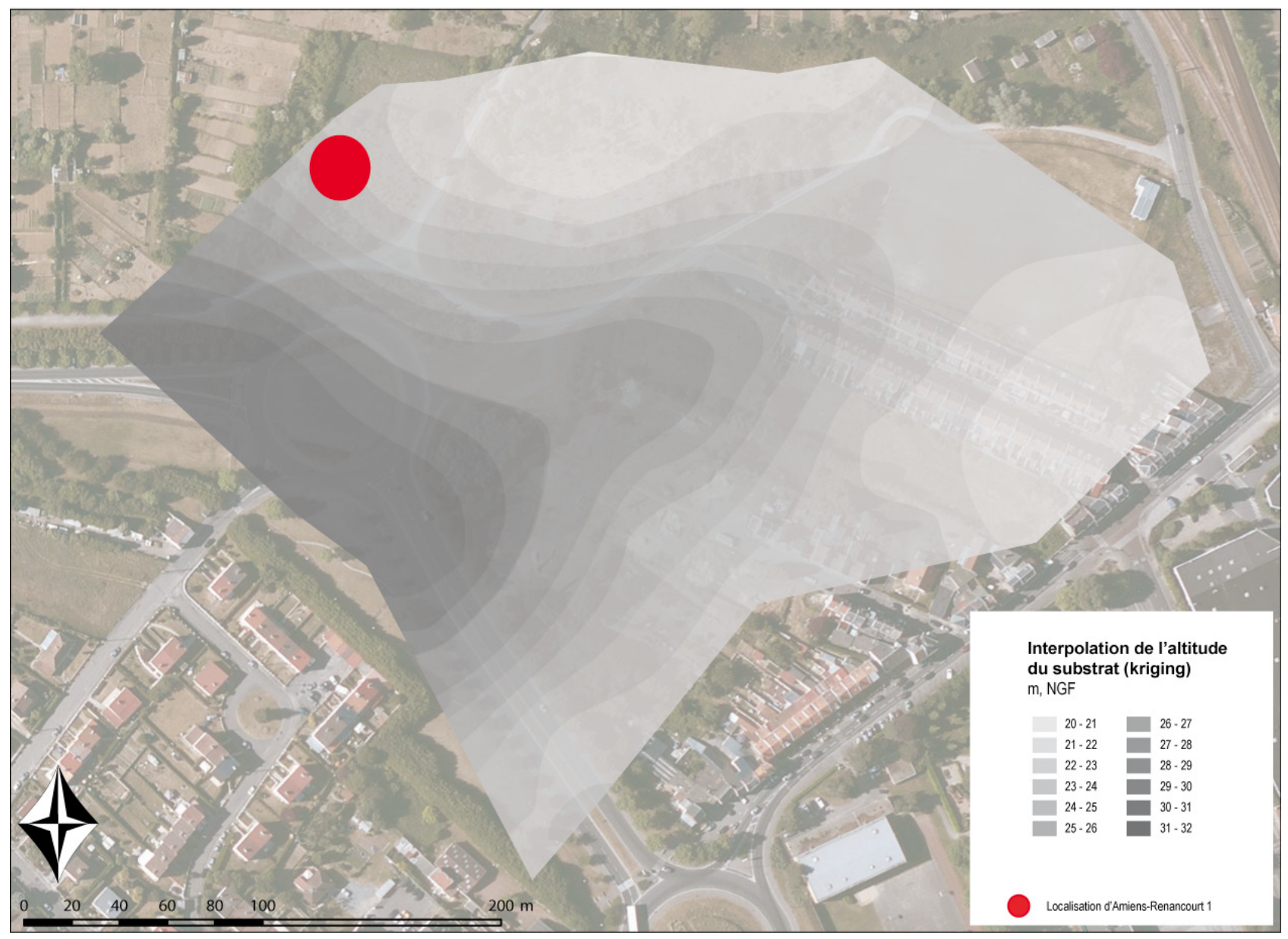

Fig. 2 - Modélisation de l'altitude du substrat à partir des données des nombreux sondages profonds réalisés sur le versant depuis 2007. En rouge : le gisement d'Amiens-Renancourt 1 (C. Font, INRAP).

Fig. 2 - Modelling of the altitude of the substratum based on data from a series of deep test pits excavated on the slope since 2007. In red: the site of Amiens-Renancourt 1 (C. Font, INRAP). 

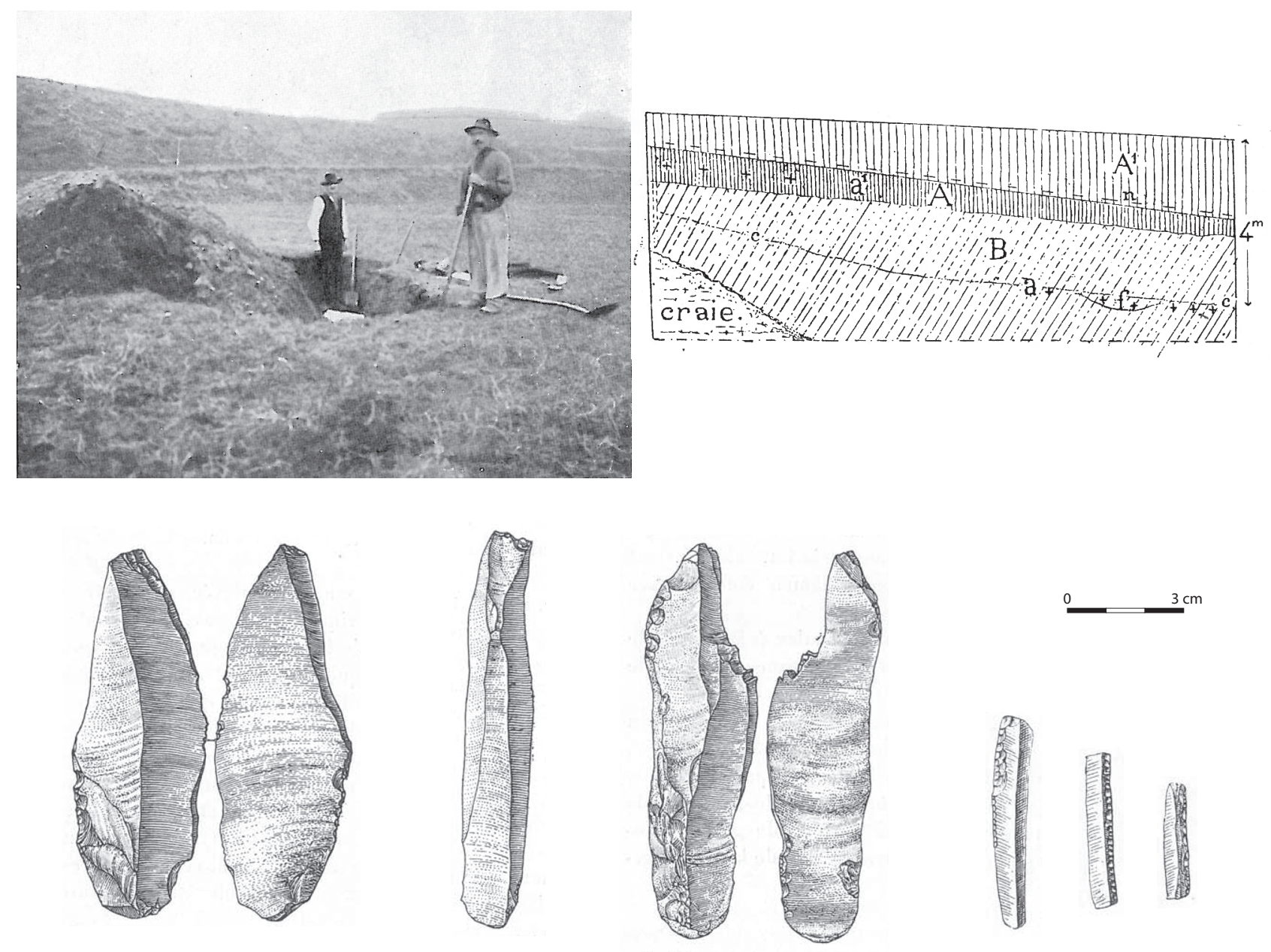

Fig. 3 - Gisement de l'ancienne briqueterie Devalois à Renancourt (Somme) découvert par Commont (1913). En haut à gauche : fouille $\mathrm{du}$ fond de l'ancienne briqueterie en 1910. En haut à droite : coupe stratigraphique de V. Commont $\left(\mathrm{A}^{1}\right.$ : limon de lavage rouge brun exploité comme terre à briques, ép. 1,50 à $2 \mathrm{~m}$; A : limon plus rouge et plus compact constituant le lehm d'altération du loess sousjacent, ép. $1 \mathrm{~m}$; B : lœss jaune chamois, très fin, calcaire, ép. 1,50 à $2 \mathrm{~m}$; c : très fin cailloutis de silex éclatés qui prend naissance sur la craie à quelque distance, ép. 0,01 à $0,05 \mathrm{~m}$. En bas : industrie lithique recueillie par Commont lors de la fouille de 1910.

Fig. 3 - Site of the 'ancienne briqueterie Devalois' at Renancourt (Somme) discovered by V. Commont (1913). Top left: excavation of the back of the brickyard in 1910. Top right: stratigraphic section of V. Commont ( $A^{1}$ : brown-red silt used as brick earth, thickness 1.50 to $2 \mathrm{~m}$; A: redder more compact silt forming the weathered lehm of the underlying loess, thickness $1 \mathrm{~m}$; $B$ : very fine fawn/buff yellow calcareous loess, thickness 1.50 to $2 \mathrm{~m}$; $\mathrm{c}$ : very fine shattered flint gravel forming on the chalk some distance away, thickness 0.01 to 0.05 m. Bottom: lithic industry collected by Commont during the 1910 excavation.

silex gélifractés, à la base d'un limon fin et calcaire altéré à son sommet (horizon B textural). Le mobilier archéologique est composé de vestiges lithiques et fauniques concentrés sur une aire restreinte de $7 \mathrm{~m}$ sur $3 \mathrm{~m}$. Un amas charbonneux d'une épaisseur de 3 à $4 \mathrm{~cm}$ contenant de l'industrie lithique chauffée (nucléus et lames) est signalé au sein de cette concentration (Commont, 1913).

Après le décès de V. Commont en 1918, la collection a malheureusement été dispersée. Notre connaissance de la série se limite donc à la publication originale de 1913, particulièrement remarquable pour l'époque par sa qualité iconographique et descriptive (fig. 3). Dans cet ouvrage, l'industrie lithique est décrite comme fortement laminaire. La plupart des artefacts, aux arêtes vives, sont non patinés et réalisés dans un silex de bonne qualité. L'auteur décompte quelques centaines d'éclats, une centaine de lames et douze nucléus. Les outils repré- sentés sont de bonne facture avec des burins relativement abondants, quelques grattoirs, des lames retouchées et cinq exemplaires de lamelles à dos dont certaines ont été réinterprétées par la suite comme des chutes de burin (Fagnart, 1988). À partir de quelques vestiges osseux, au moins trois espèces animales ont été déterminées : le cheval (maxillaire inférieur), le bovidé (métatarsien de bison?) et le cervidé (extrémité d'un bois). Jusqu'à une date récente, ce gisement représentait pour le nord de la France le seul témoignage quantitativement important du Paléolithique supérieur ancien (plusieurs centaines de pièces) contenu dans un contexte stratigraphique fiable.

Malgré l'intérêt de cette découverte, il faudra attendre les travaux de l'un d'entre nous (J.-P. F.) dans les années 1980 pour intégrer ce site dans une synthèse régionale et proposer une attribution au « Périgordien supérieur » sur une base typologique (Fagnart, 1980 et 1988). 
En 1996 et 1997, deux des auteurs (J.-P. F. et P. C.) ont l'opportunité de sonder les abords de l'ancienne briqueterie Devalois dans le cadre d'un diagnostic archéologique lié à la réalisation de la pénétrante ouest d'Amiens (Fagnart et Coudret, 1997). Quelques silex taillés à tendance laminaire et des fragments de faune sont recueillis. Ces découvertes sont alors considérées comme le témoignage des marges du gisement étudié par Commont. Les quelques vestiges mis au jour ne sont pas très caractéristiques et certains se situent en position secondaire, notamment dans le bas du versant. Deux datations ont pu être obtenues sur des ossements attribués au cheval; elles placent l'occupation archéologique dans la dernière partie du complexe Gravettien, entre 22000 et 23000 BP non cal. (Fagnart et al., 2013). Néanmoins, aucun élément typotechnologique significatif n'a pu être collecté compte tenu de la faiblesse du corpus mais un rapprochement avec la phase récente du Gravettien a été prudemment avancé sur la base des dessins de Commont (Fagnart et al., 2013).

Plus récemment, la création de la ZAC de Renancourt sur une surface de plusieurs dizaines d'hectares a permis d'entreprendre de nouvelles opérations archéologiques dans ce secteur. Les différentes phases de diagnostic, menées de 2007 à 2011, apportent de nombreuses informations avec la découverte d'une zone inédite, Amiens-Renancourt 2, qui s'étend sur une surface de près de $5000 \mathrm{~m}^{2}$ le long du versant de la vallée de la Selle, à environ $200 \mathrm{~m}$ au sud-est des précédentes découvertes (fig. 1). Près de $1800 \mathrm{~m}^{2}$ de ce gisement ont été explorés dans le cadre de deux fouilles préventives (Paris, 2015). Elles livrent plusieurs concentrations peu denses de vestiges lithiques et fauniques, correspondant à des occupations brèves, spécialisées dans des activités de taille et de boucherie. L'occupation datée vers 28000 BP non cal. est incluse dans une séquence stratigraphique attribuable à la fin du Pléniglaciaire moyen (Paris et al., à paraître).

Une seconde zone présentée dans le cadre de cet article, est dénommée Amiens-Renancourt 1 (fig. 1). Révélée par un sondage profond en 2011, elle se situe à proximité immédiate de l'ancienne briqueterie Devalois et des opérations de terrain réalisées en 1996 et 1997. Les artefacts découverts à une profondeur de $4 \mathrm{~m}$ ont été uniquement récupérés dans le godet de la pelle mécanique pour des raisons de sécurité (Paris et al., 2013). L'industrie récoltée évoque de manière évidente celle mise au jour par $\mathrm{V}$. Commont au début du $\mathrm{Xx}^{\mathrm{e}}$ siècle. La fraîcheur des bords des artefacts, l'état de conservation de l'ensemble des vestiges et l'évidence d'avoir redécouvert le niveau archéologique étudié par V. Commont avant la première guerre mondiale nous ont incités à poursuivre les investigations sur cette nouvelle concentration.

\section{LE GISEMENT D'AMIENS-RENANCOURT 1}

D epuis 2013, des opérations programmées annuelles ont permis d'enrichir considérablement les observations du sondage profond. Aujourd'hui, $41 \mathrm{~m}^{2}$ ont été totalement fouillés, mais l'étendue présumée de la nappe de vestiges n'est pas encore connue. Plusieurs centaines de mètres carrés restent encore à explorer entre « l'ancienne briqueterie Devalois » et la fouille actuelle.

\section{Stratigraphie}

Pour le gisement de Renancourt 1, deux levés stratigraphiques ont été jusqu'ici publiés. Le premier provient des sondages effectués en 1996 et 1997 où quelques rares vestiges ont été découverts dans les limons lités du Pléniglaciaire supérieur situés sous l'horizon à langues de Nagelbeek (Fagnart et al., 2013). Le deuxième a été publié à partir du sondage profond de 2011 qui a révélé le gisement (Paris et al., 2013). Là encore, le niveau archéologique a été localisé dans un épais dépôt de limons lités attribué au Pléniglaciaire supérieur. Si dans un premier temps la position des vestiges semblait cohérente, les opérations menées depuis 2013 montrent cependant l'inexactitude des premières observations et précisent la localisation réelle du niveau archéologique. La séquence stratigraphique actuelle, beaucoup plus complète, situe l'occupation paléolithique dans un gley de toundra clairement distinct des dépôts de limons lités sus-jacents (fig. 4). Cette opposition entre les premières observations et les recherches actuelles s'explique par des raisons différentes :

1) Pour les opérations réalisées en 1996 et 1997, les quelques pièces mises au jour dans les limons lités proviennent de sondages situés plus bas sur le versant. Cette position topographique, ainsi qu'une patine et une éolisation fortes des témoins lithiques vont dans le sens d'une remobilisation des pièces lors du dépôt des limons lités. Certains de ces artefacts avaient d'ailleurs déjà été considérés en position secondaire (Fagnart et Coudret, 1997).

2) En ce qui concerne le sondage profond de 2011, il s'agit d'une erreur liée à la méthodologie inhérente aux investigations en puits. Lors de la réalisation de sondages à forte profondeur, les observations géologiques sont en effet réalisées directement dans le godet de la pelle mécanique. Avec cette méthode, il est souvent difficile de faire une distinction stratigraphique fine entre les différentes unités rencontrées, plus particulièrement lorsque les faciès sédimentaires sont peu contrastés. De ce fait, aucune différence n'a été perçue dans le godet entre les limons lités, les lœss homogènes sous-jacents et le gley contenant l'industrie.

Un nouveau levé, réalisé dans des conditions optimales, permet aujourd'hui de préciser l'emplacement de l'occupation archéologique au sein de l'épaisse séquence stratigraphique (env. $8 \mathrm{~m}$ ). Cette dernière est constituée, du sommet vers la base, des unités suivantes (fig. 4) :

0 - Limon brun gris à structure granulaire, silex et fragments de brique épars, limite inférieure franche (horizon Ap du sol de surface);

1 - Limon argileux non calcaire, modérément compact, brun-rouge à traces de racines, nombreux biotubules de vers de terre et grosses bioturbations (galeries) remplies de matériel en provenance de la surface ou du 


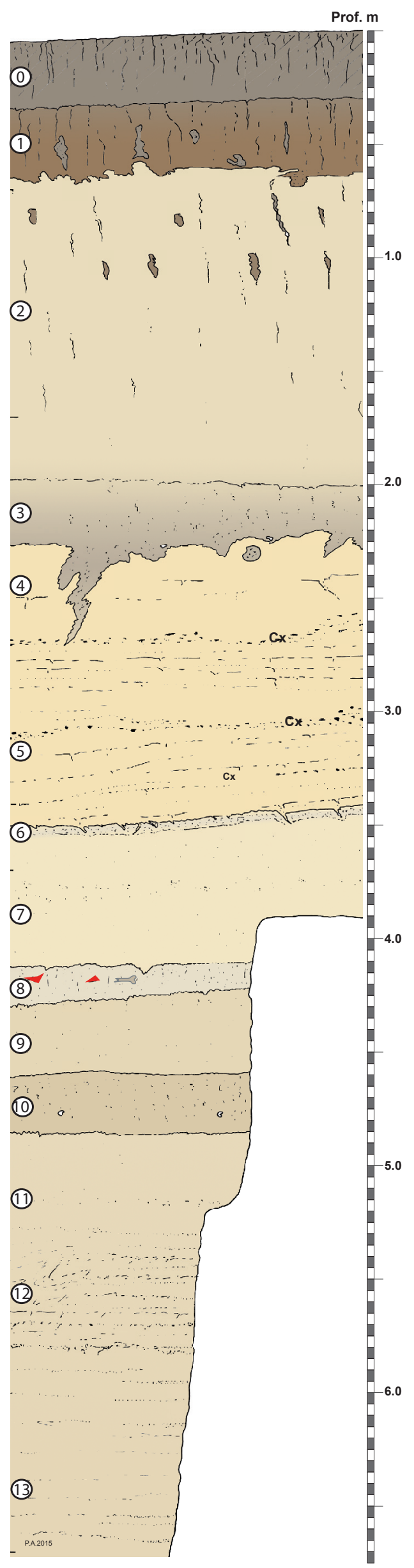

Fig. 4 - Coupe stratigraphique du gisement d'Amiens-Renancourt 1 (P. Antoine, CNRS).

Fig. 4 - Stratigraphic section of the site of Amiens-Renancourt 1 (P. Antoine, CNRS).

lœss sous-jacent. Limite basale irrégulière (bioturbation) (horizon Bt tronqué du sol de surface sur lœess);

2 - Lœss calcaire sableux homogène beige grisâtre clair à très nombreux tubules de carbonates secondaires de grande taille (rhyzolithes), nombreuses galeries de vers subverticales à colmatage argilo-humique dans la moitié supérieure (lœss calcaire homogène typique). Limite inférieure très diffuse et progressive avec l'horizon 3 ;

3 - Horizon loessique homogène gris brunâtre (légèrement humifère) avec très abondants carbonates secondaires (tubules ø 2-5 $\mathrm{mm}$ ), pseudomycelium et quelques concrétions calcaires centimétriques à la base. Limite inférieure assez nette mais très déformée (horizon à langues de gélifluxion). Localement présence d'une fente d'environ $0,5 \mathrm{~m}$ de profondeur fortement déformée (horizon de type « horizon à langues de Nagelbeek »);

4 - Lœss calcaire sableux brun-jaune clair à rares litages sableux et microfentes de cryodessiccation, petites taches noires ferromanganiques éparses. Limite inférieure nette, marquée par un cailloutis de petits silex gélifractés $(\leq 1 \mathrm{~cm})$ et de granules de craie;

5 - Lœss calcaire sableux compact brun-jaune à lits gris clair millimétriques et microfentes de cryodessiccation bien marquées (loess calcaires lités sableux à microfentes). Un cailloutis discontinu de silex gélifractés et de granules de craie scinde ce dépôt en deux parties. Limite inférieure nette avec l'unité 6 associée à des microfentes;

6 - Petit horizon loessique grisâtre à taches et linéoles oxydées orangées et concrétions ferromanganiques à la base. Limite inférieure diffuse avec le loess 7 ;

7 - Lœss calcaire homogène gris-beige à pseudomycelium épars. Présence de petits horizons diffus à taches d'oxydation dans la partie médiane;

8 - Horizon lœssique décimétrique à taches grisâtres et linéoles oxydées orangées (racines) et concrétions ferromanganiques à la base. Limite inférieure irrégulière avec le loss 9 (gley de toundra). Le niveau paléolithique se situe dans la partie médiane à supérieure de cet horizon;

9 - Lœess calcaire homogène dense beige à gris clair à concrétions ferromanganiques éparses $(<1 \mathrm{~mm})$;

10 - Lœss calcaire compact homogène brunâtre à brun grisâtre avec granules de craie et petits silex gélifractés épars. Pseudomycelium abondant et nombreuses précipitations ferromanganiques au sommet. Limite inférieure diffuse. Latéralement, cet horizon devient plus hydromorphe avec un faciès de gley de toundra;

11 - Lœss homogène compact brun clair à rares granules de craie épars et quelques pseudomycelium. Limite inférieure diffuse passage à un faciès à fins lits sableux;

12 - Lœss sableux lités à lits grisâtres et orangés avec déformations assez marquées (gélifluxion). Ensemble assez riche en lits de granules de craie et de petits silex 
gélifractés épars $(\sim 5 \mathrm{~mm})$. Faciès nettement ruisselé avec incorporation d'éléments érodés sur le versant;

13 - Ensemble de lœss sableux ruisselés, compacts à litages sableux et rares granules de craie (base non atteinte).

Un sondage complémentaire à la tarière, mené à partir de la base de ce profil, a rencontré sur 1,70 m des limons de faciès semblable incluant de plus en plus de granules de craie et de silex gélifractés dans les derniers $40 \mathrm{~cm}$. La base de cette unité limoneuse repose sur un cailloutis compact de silex gélifractés et blocs de craie qu'il n'a pas été possible de traverser.

La séquence de Renancourt s'intègre parfaitement dans le cadre des enregistrements décrits pour le Pléniglaciaire supérieur du Weichselien, caractérisés par une accélération des taux de sédimentation lœssique (Antoine et al., 2016). Il convient de noter l'absence de grands coins de glace qui caractérisent plus particulièrement les ensembles stratigraphiques développés en position de plateau. L'horizon repère de la partie supérieure de la stratigraphie (unité 3) est considéré comme l'équivalent de l'« horizon à langues de Nagelbeek » qui sépare habituellement les lœss calcaires homogènes typiques (unité 2) des lœss calcaires lités à microfentes (unités 4 et 5). La partie inférieure de la séquence est composée par un ensemble de lœss calcaires homogènes alternant avec un gley de toundra peu épais (unité 8) et un horizon loessique brunifié-gley de toundra (unité 10). L'occupation gravettienne se place dans le gley de toundra supérieur (unité 8). Cette succession de lœss calcaires homogènes n'avait pas encore été observée dans la région d'Amiens. Les deux horizons pédogénétiques intercalés peuvent être rapprochés d'un point de vue chronostratigraphique de l'« horizon cryoturbé du Santerre » identifié dans l'est de la Somme (Antoine, 1991) et des gleys de toundra superposés du sommet de la séquence d'Havrincourt dans le Pas-de-Calais (unités 3a et 3c; Antoine et al., 2014). Dans la séquence de référence de Nussloch (Allemagne) située dans la vallée du Rhin vers Heidelberg, le doublet de gleys de toundra équivalent (G3 et G4; Antoine et al., 2009) est daté entre environ 29 et 27 ka (soit 23000 à 25000 BP non cal.).

L'unité 8 contenant l'industrie se place donc clairement avant le dépôt des loess laminés à microfentes, qui correspond à l'épisode de sédimentation le plus épais dans la région. D'après les données malacologiques préliminaires (O. M.), ce gley de toundra est associé à des conditions interstadiaires plus clémentes qui s'accompagnent généralement de la fonte progressive du pergélisol et de l'engorgement de la couche active à chaque dégel saisonnier. D'après les enregistrements climatiques des carottes de glace du Groenland (Rasmussen et al., 2014), il serait contemporain de l'interstade GI 3. L'occupation se place dans la première partie de cette phase, dans un contexte de transition stade-interstade caractérisé par un environnement steppique largement ouvert.

\section{Conditions de conservation du gisement}

Jusqu'à une date récente, les observations menées lors de la fouille s'orientaient davantage vers l'existence d'un niveau d'occupation ayant subi peu ou pas de déplacements, à la suite d'un enfouissement rapide par des dépôts éoliens. Les conditions de préservation des témoins archéologiques apparaissaient relativement bonnes bien qu'une légère pente puisse atteindre $10 \%$ par endroit (fig. 5). Les premiers tests de fabriques réalisés par P. Bertran
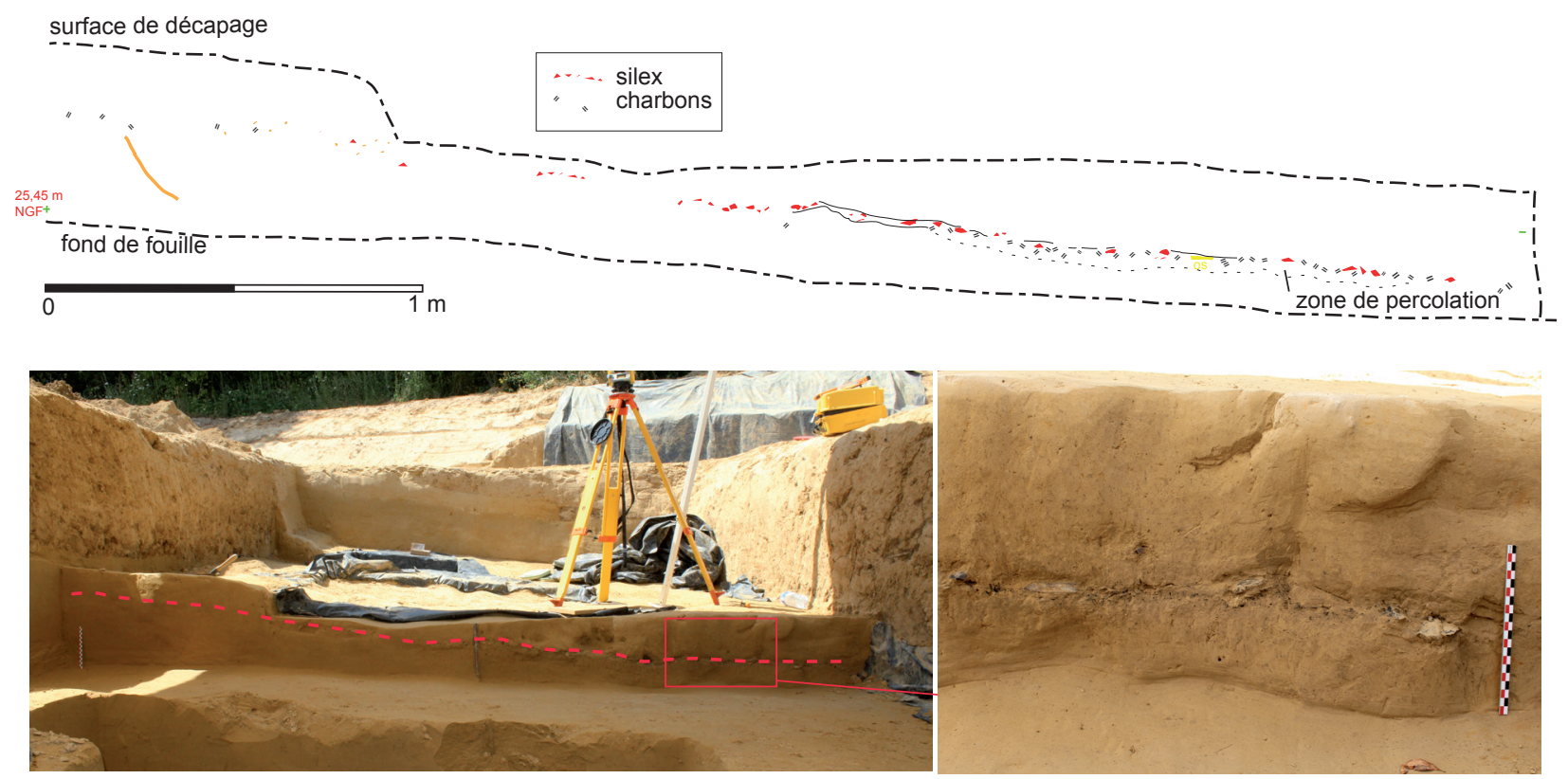

Fig. 5 - Amiens-Renancourt 1 (Somme), levé et clichés de la coupe nord-sud de la partie fouillée en 2014 et 2015 (S. Coutard, INRAP). Fig. 5 - Amiens-Renancourt 1 (Somme), drawing and photos of the north-south section in the zone excavated in 2014 and 2015 (S. Coutard, INRAP). 
ont cependant montré que le niveau archéologique a été affecté à certains endroits par des déplacements (cryoreptation, solifluxion) en milieu périglaciaire. Les déformations du niveau archéologique restent néanmoins limitées. Elles suggèrent que l'organisation initiale de l'occupation humaine n'a été que partiellement modifiée par les processus naturels postérieurs à l'abandon du site. Il est fortement probable que l'identification des zones d'activités à partir de la distribution des vestiges reste tout à fait lisible, ce que confirment d'ailleurs les observations archéologiques avec une répartition différentielle de certaines catégories de vestiges (parures, outils par exemple). D'une manière générale, ce gisement, qui est de peu antérieur au Dernier Maximum Glaciaire, apparaît bien conservé par comparaison avec d'autres sites connus pour cette période, vraisemblablement en raison d'un enfouissement rapide par des dépôts de lœss poudreux.

\section{Datations de l'occupation gravettienne}

Quatre datations ${ }^{14} \mathrm{C}$ ont été réalisées sur os, os chauffé et charbon non déterminés. Elles viennent s'ajouter aux deux datations réalisées sur des ossements de chevaux découverts en 1997 (Fagnart et al., 2013). Ces six dates sont très cohérentes entre elles et placent chronologiquement le niveau archéologique entre 22000 et 23000 BP, soit aux alentours de 27000 cal. BP (tabl. 1).

Deux datations par luminescence optiquement stimulée (OSL) sur quartz (fraction 5-11 $\mu \mathrm{m}$ ) ont également été réalisées dans le gley de toundra contenant le niveau archéologique (tabl. 2). Là encore, les résultats attribuent au niveau paléolithique un âge moyen de $27500 \pm 1500$ ans. Ces mesures d'âges sont en accord avec les datations ${ }^{14} \mathrm{C}$ calibrées en tenant compte de l'écart-type de 1500 ans. Autrement dit, en raison de la superposition des marges d'incertitude, le fait qu'elles soient légèrement plus anciennes n'est pas significatif.

\section{Aperçu général de la documentation archéologique}

Dégagé sur près de $41 \mathrm{~m}^{2}$, le niveau archéologique a livré plus de 7500 éléments lithiques et osseux et plus de 50000 éléments de très petites dimensions récoltés au tamisage à sec. À cet ensemble s'ajoutent de l'art mobilier, des éléments de parure et quelques rares fragments d'industrie osseuse. Au regard de la surface fouillée, il est encore difficile de cerner l'organisation et les limites de l'occupation (fig. 6). Une zone très dense de près de $10 \mathrm{~m}^{2}$ résulte de l'accumulation de vestiges de nature diverse (rejets), pouvant atteindre près de 900 éléments au mètre carré. Dans cet espace, la présence de restes fauniques brûlés et de nombreux charbons, souvent associés en petits dépôts, et de quelques grès altérés thermiquement pourraient également résulter de vidanges de foyers. À l'ouest de cette zone, se développe une nappe de vestiges plus épars au sein de laquelle s'individualisent des zones d'activités, sous la forme de petits amas, liées au travail du silex et d'autres plus spécifiquement dédiées à la fabrication de statuettes et d'éléments de parure en craie.

L'industrie lithique est caractérisée par une production de lames de grand gabarit. Les blocs de matière première sont issus des formations de la craie du Turonien supérieur ou du Coniacien basal qui affleurent localement dans la vallée de Grâce à quelques dizaines de mètres de la concentration. Ce silex noir avec un cortex de couleur jaune clair ou plus souvent rosée offre de très bonne aptitude à la taille et se présente sous la forme de rognons de grandes dimensions permettant d'obtenir des lames dépassant parfois les $20 \mathrm{~cm}$. La mise en forme des blocs

\begin{tabular}{|l|l|c|c|l|}
\hline Référence de l'échantillon & Matériau & $\hat{A g e ~}{ }^{14} \mathrm{C}$ BP & $\hat{A} g e$ calibré $(2 \sigma ;$ IntCal 13) & Références \\
\hline Lyon-9943 (SacA 32189) & Os & $22600 \pm 170$ & $26473-27336$ cal. BP & Inédit \\
\hline Lyon-9942 (SacA 32188) & Os chauffé & $23580 \pm 180$ & $27423-28008$ cal. BP & Inédit \\
\hline Beta-306063 & Os & $21890 \pm 90$ & $25899-26345$ cal. BP & Paris et al., 2013 \\
\hline Lyon-11659 (SacA 39279) & Charbon & $23250 \pm 210$ & $27805-27165$ cal. BP & Inédit \\
\hline Lyon-632 & Os & $23040 \pm 220$ & $26894-27730$ cal. BP & Fagnart et al., 2013 \\
\hline Lyon-633 & Os & $22360 \pm 240$ & $26096-27217$ cal. BP & Fagnart et al., 2013 \\
\hline
\end{tabular}

Tabl. 1 - Datations ${ }^{14} \mathrm{C}$ d'Amiens-Renancourt 1. En haut : celles obtenues depuis 2011; en bas : celles réalisées à l'issue des sondages de 1997 (calibration avec OxCal, d'après Reimer et al., 2013).

Table 1 - Radiocarbon dates from Amiens-Renancourt 1. Top: dates obtained since 2011; bottom: dates obtained during the 1997 test pits (calibration with OxCal, after Reimer et al., 2013).

\begin{tabular}{|l|l|l|l|}
\hline Référence de l'échantillon & Localisation & Date & Références \\
\hline OSL-2014-4 & Gley & $27200 \pm 1500$ & inédit G. Guérin \\
\hline OSL-2013-1 & Gley & $27900 \pm 1500$ & inédit G. Guérin \\
\hline
\end{tabular}

Tabl. 2 - Datations OSL sur sédiment de l'unité 8 contenant le niveau archéologique.

Table 2 - OSL dates on sediment from unit 8 containing the archaeological level. 


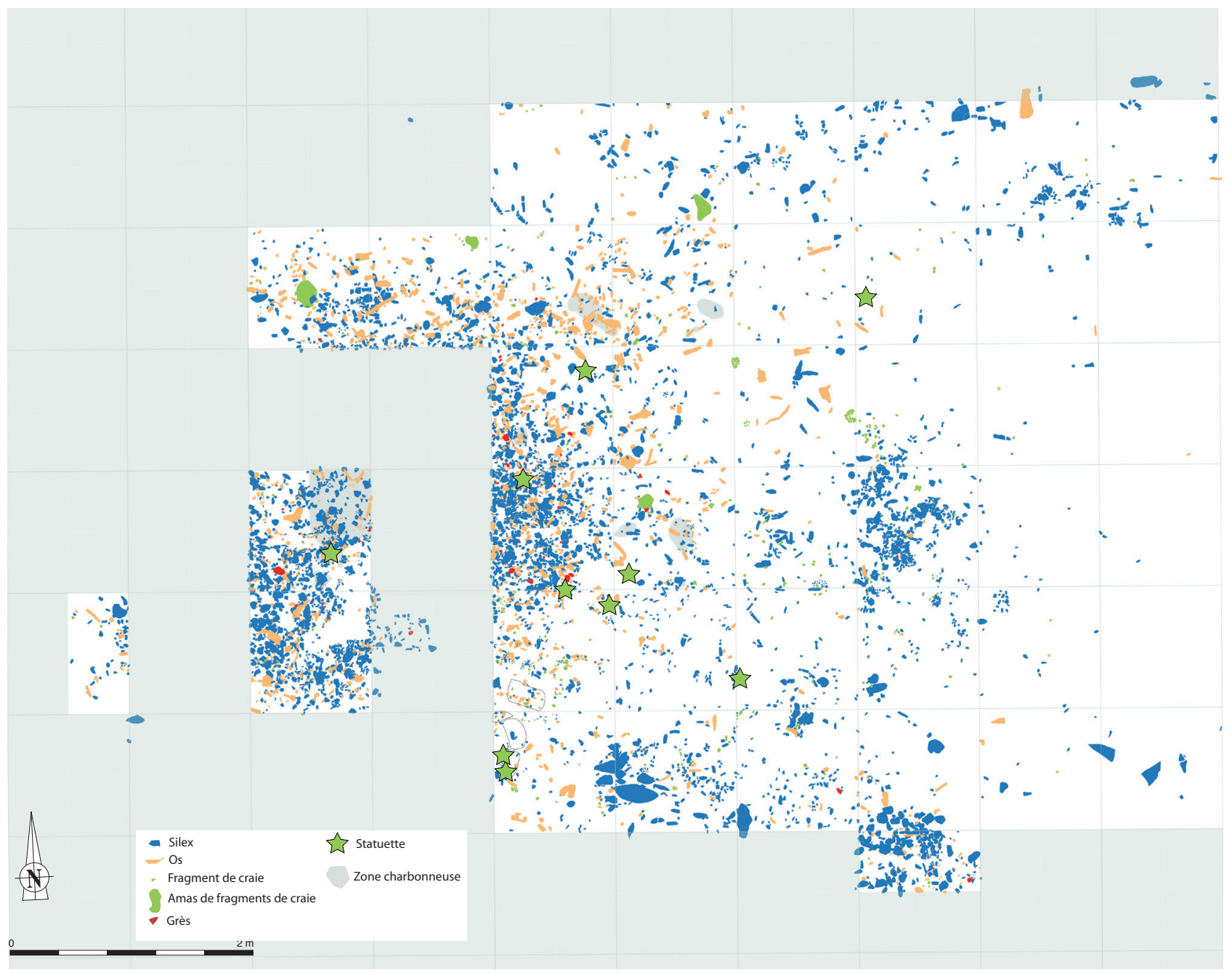

Fig. 6 - Amiens-Renancourt 1 (Somme). Répartition spatiale des vestiges découverts lors des campagnes de fouilles de 2013 à 2016.

Fig. 6 - Amiens-Renancourt 1 (Somme). Spatial distribution of the remains discovered during the excavations conducted from 2013 to 2016 .

est soignée, avec l'installation d'une crête médiane antérieure et d'une ou deux crêtes postérieures. La préparation au détachement fait également l'objet d'une attention particulière avec la réalisation d'éperons. Ceux-ci évoquent, en plus d'autres stigmates (lèvre bien marquée, ligne postérieure du talon nette et régulière, bulbe diffus, talon nettement mesurable), l'utilisation d'un percuteur tendre organique (Pelegrin, 2000). Cette production est dévolue à la fabrication de l'outillage commun (burins, grattoirs...) et de quelques armatures de grandes dimensions (pointes de la Gravette). Parallèlement, une production lamellaire autonome est réalisée sur la tranche de gros éclats, plus rarement sur des lames épaisses. Elle a pour objectif la fabrication d'armatures dont le corpus, d'une cinquantaine d'éléments, est largement dominé par les fragments mésiaux de pièces à dos lamellaires auxquelles il faut ajouter quelques lamelles à retouche marginale et quatre microgravettes (fig. 7). Il est intéressant de noter parmi ces lamelles, la découverte d'une dizaine de produits en silex tertiaire exogène. L'examen à la loupe binoculaire a permis la reconnaissance d'Oogones de characées qui orientent vers un silex de formation lacustre, très probablement de l'étage Bartonien. Les premiers affleurements se situent en rive droite de l'Oise, au niveau de la ville de Creil, soit à $70 \mathrm{~km}$ environ à vol d'oiseau du site d'Amiens-Renancourt 1 (fig. 1).

La faune est assez bien conservée comme en témoigne la présence de plusieurs ossements de fœtus, malgré une dégradation des surfaces osseuses liées au contexte de plein air. Avec plus de 1700 restes enregistrés, le spectre faunique est dominé de manière écrasante par le cheval. Quelques fragments pourraient cependant appartenir à un cervidé de la taille du renne ou du cerf. Un fragment d'ivoire transformé en outil (étude N. Goutas), attribué à un proboscidien, ainsi qu'une mandibule de lièvre complètent la liste faunique. Les premières indications sur la saisonnalité à partir des os de fœutus et de jeunes suggèrent une occupation durant la belle saison, entre le début du printemps et l'automne. Le site apparaît d'ores et déjà comme un lieu hautement stratégique pour la chasse. 


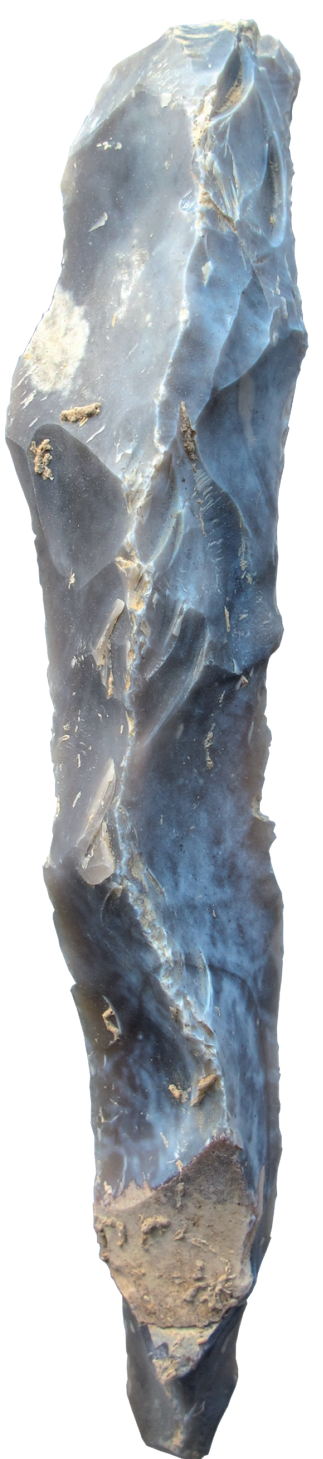

1

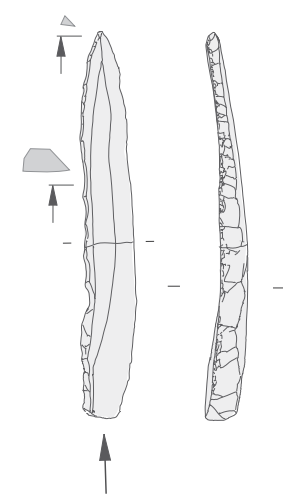

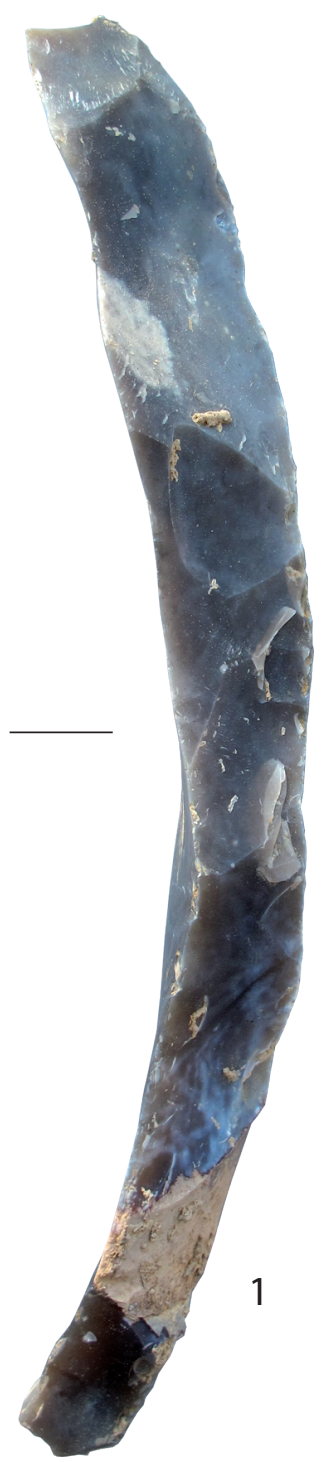
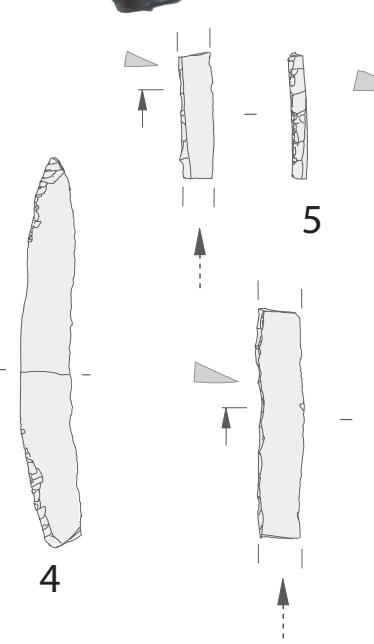
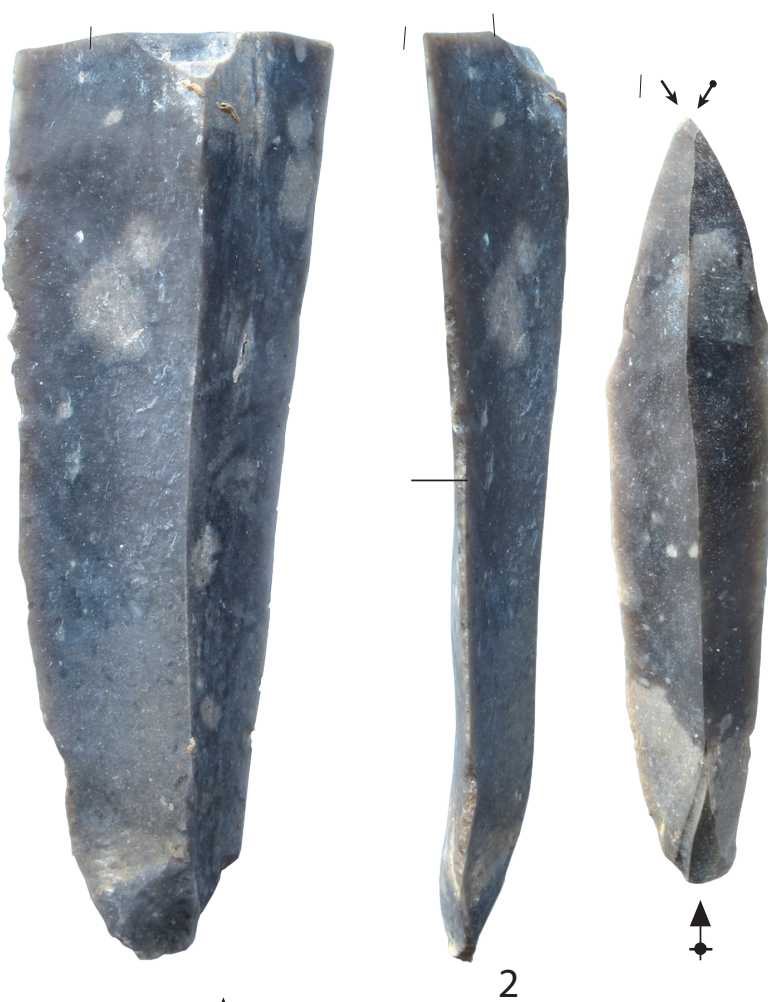

$\downarrow$

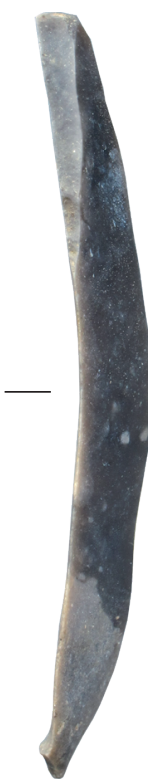

3

Fig. 7 - Industrie lithique d'Amiens-Renancourt 1.1 : lame à crête; $2:$ fragment proximal de lame de plein débitage; $3:$ burin dièdre sur petite lame; $4:$ microgravette; 5 à $7:$ lamelles à dos : $8:$ pointe de la Gravette (clichés S. Lancelot, INRAP et dessins J. Airvaux).

Fig. 7 - Lithic industry from Amiens-Renancourt 1. 1: crested blade; 2: proximal fragment of a blade from the full knapping phase; 3: dihedral burin on small blade; 4: microgravette; 5 to 7: backed bladelets: 8: Gravettian point (photos S. Lancelot, INRAP and drawings J. Airvaux). 


\section{LES PARURES}

$\mathrm{L}$ es éléments assimilés à de la parure comprennent vingt et une pièces. Ils se répartissent en deux catégories principales : des fragments de rondelles en craie $(\mathrm{n}=11)$ et des coquillages fossiles (moules internes de Turritelles, $n=10$ ). Bien que ces dernières ne présentent pas de modification anthropique, leurs caractéristiques esthétiques et dimensionnelles ainsi que leurs origines exogènes (voir ci-dessous) nous incitent à les classer dans la catégorie des parures. Des éléments similaires en Belgique, dans des niveaux attribués au Magdalénien, ont d'ailleurs été considérés comme tels (Moreau, 2003).

\section{Les rondelles en craie}

Parmi les onze fragments de rondelles, trois éléments découverts à proximité les uns des autres raccordent entre eux et forment un exemplaire entier (fig. 8, $\mathrm{n}^{\circ} 1$ ). Les autres fragments représentent dans un cas la moitié d'une rondelle (fig. $8, \mathrm{n}^{\circ} 2$ ) et dans les autres cas moins d'un quart d'une rondelle (fig. $8, \mathrm{n}^{\text {os }} 3$ à 9 ). Ils proviennent tous de rondelles d'environ $30 \mathrm{~mm}$ de diamètre à perforation centrale et à profil plano-convexe. La rondelle complète porte une série de treize " crans » transversaux, parallèles et régulièrement espacés positionnés sur le pourtour (fig. 8, $\mathrm{n}^{\circ} 1 \mathrm{a}$ ). Ils sont réalisés par sciage avec plusieurs va-et-vient à l'aide d'un tranchant lithique. Ce motif est également présent sur un autre fragment qui porte une série de huit « crans » plus fins et moins marqués (fig. 8, $n^{\circ} 3 b$ ). Les autres fragments ont des bords lisses et arrondis qui sont entièrement ou en partie régularisés par abrasion. Les perforations sont observables sur les fragments de l'exemplaire reconstitué dans sa globalité et sur celui représentant une demi-rondelle. S'ajoutent de possibles traces de perforation sur deux autres fragments. Les perforations ont été réalisées par piquetage ou percussion indirecte depuis les deux faces sur l'exemplaire entier (section biconique : fig. $8, \mathrm{n}^{\circ} 1 \mathrm{c}$ ) et depuis une seule face sur la demi-rondelle (section conique : fig. $8, \mathrm{n}^{\circ} 2 \mathrm{a}$ ). La perforation de la demi-rondelle n'est pas aboutie, celleci n'a en effet pas traversé toute l'épaisseur de la pièce. Enfin, il est à noter que la plupart des fragments présentent sur une ou deux faces des plages de stries résultant du façonnage des pièces.

Plusieurs de ces observations suggèrent une production sur place de ces objets. Ces fragments de rondelles paraissent être des ébauches. La perforation non aboutie de la demi-rondelle en est le principal argument, auquel s'ajoute la présence de surfaces striées qui n'ont pas été régularisées et des nombreuses fractures qui, si elles sont intervenues en cours de fabrication, expliquent que ces pièces aient été abandonnées en l'état.

\section{Les moules internes de Turritelles}

La seconde catégorie d'éléments de parure comprend dix moules internes épigénisés de gastéropodes fossiles (fig. 8, $\mathrm{n}^{\text {os }} 10$ à 19). Huit ont été découverts dans un espace restreint de trois quarts de mètre carré. La forme générale indique qu'il s'agit certainement de moules internes de Turritelles (les Cérithes et les Potamides, de formes proches, ont des spires plus arrondies), à silicification orangée et translucide. Ces pièces mesurent entre 15 et $25 \mathrm{~mm}$ de hauteur.

Ces fossiles ne sont pas présents dans les dépôts géologiques de la région d'Amiens. Ils caractérisent le Lutétien de la moyenne vallée de l'Oise, dont les affleurements les plus proches se situent à environ $70 \mathrm{~km}$ vers le sud-est entre Noyon et Creil. Cependant, les moules internes d'Amiens-Renancourt 1 sont assez particuliers. Leur silicification originale ne se retrouve que dans des faciès très localisés du Lutétien, à une centaine de kilomètres d'Amiens (fig. 1) dans les vallées des bassins versants de l'Ourcq (à Longpont dans l'Aisne), de l'Aisne (à Courville dans la Marne) ou encore de la Mauldre (à Maule dans les Yvelines). Ces fossiles peuvent également être trouvés en position secondaire dans des formations alluviales, mais les Turritelles d'Amiens-Renancourt 1 paraissent trop peu érodées (partie calcaire conservée) pour provenir de ce type de dépôt. Une collecte sur un gîte primaire est donc l'hypothèse privilégiée.

\section{LES STATUETTES FÉMININES EN CRAIE}

Depuis 2014, neuf rondes-bosses en craie, ainsi qu'un fragment de silex travaillé qui pourrait se rattacher à la catégorie des objets d'art mobilier, ont été mis au jour (fig. 9 à 11). Cette découverte est exceptionnelle pour l'Europe occidentale. En France notamment, la dernière trouvaille de ce type d'objet en contexte stratigraphique gravettien est la Vénus de Tursac, par H. Delporte en 1959 (Delporte, 1959).

Les sculptures, ou fragments partiellement sculptés, ont été identifiées et prélevées sur le site en tant qu'objet archéologiquement entier (à l'image des statuettes des figures 10 et 11) ou sous la forme de petits amas de fragments de craie (objets fragmentés, comme la statuette de la figure 9). Leur taille varie sensiblement, même si la majorité d'entre elles font moins de trois centimètres de hauteur (tabl. 3). La plus grande, qui est aussi la première à avoir été découverte, mesure plus de dix centimètres de haut (fig. 9). La fréquence des petits modules s'explique en partie par l'état souvent fragmentaire des sculptures :

- trois fragments correspondent à des poitrines (fig. 11a, b et e);

- un fragment représente une protubérance (tête? sexe masculin? : fig. 11d);

- deux autres sont des membres inférieurs (des hanches aux genoux) de corps féminin (fig. 10b et c);

- une statuette est acéphale (fig. 10a);

- deux d'entre elles présentent des corps entiers, bien que la plus grande ait perdu sa cuisse droite (fig. 9 et 10d).

Les plus complètes, à l'exception de l'une d'entre elles, possèdent les mêmes particularités : les caractères féminins sont prononcés, avec souvent une poitrine opulente $(n=4)$ et des fesses exagérément projetées vers 

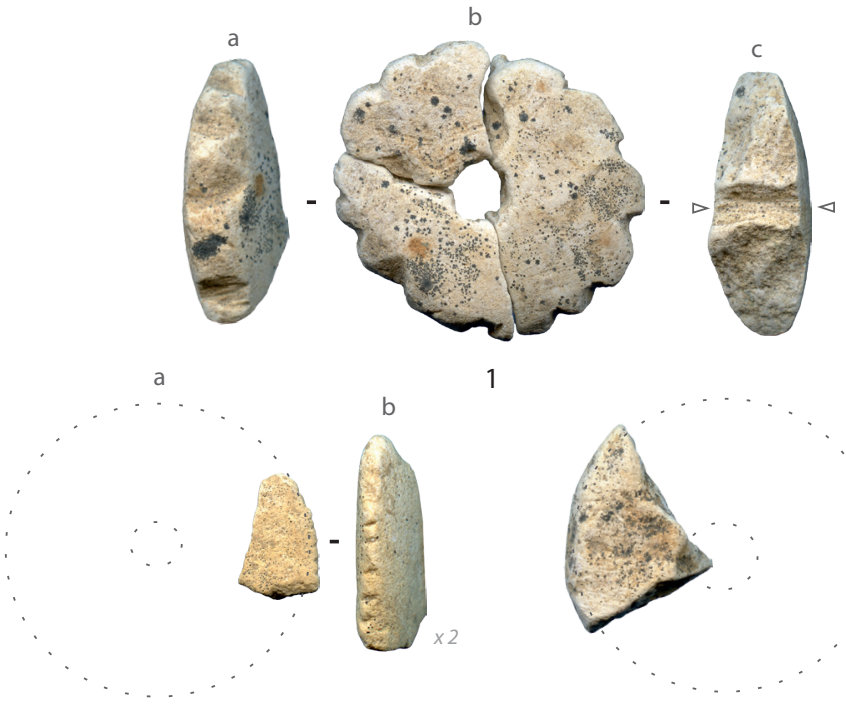

3

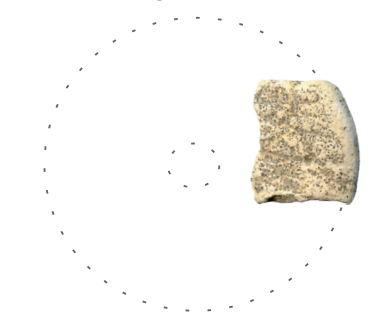

7

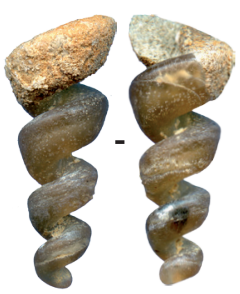

10

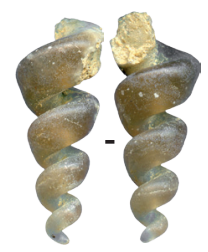

15

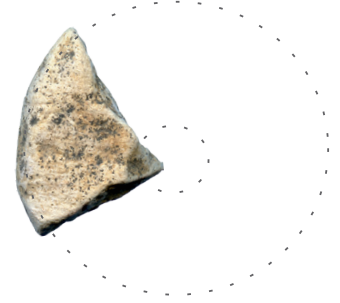

4

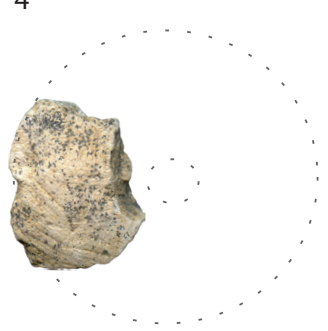

8

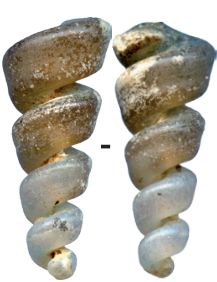

11

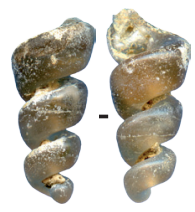

16

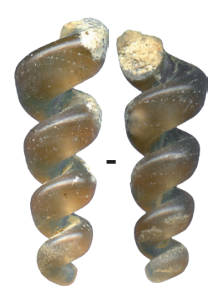

12

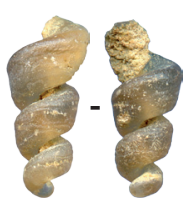

17

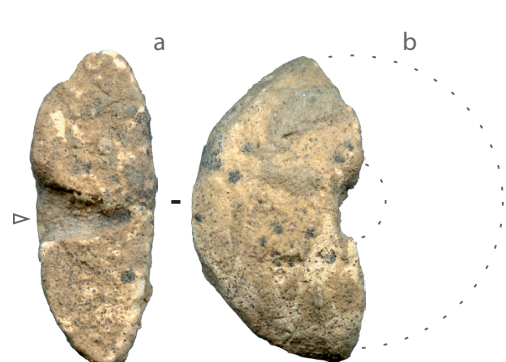

2

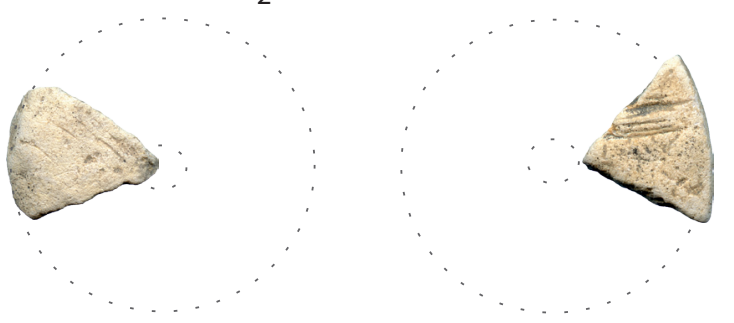

5

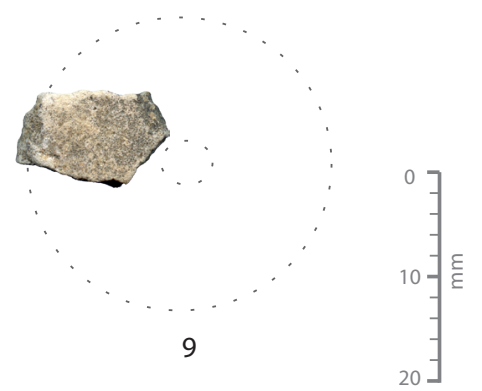

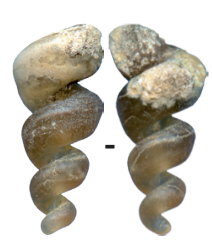

14

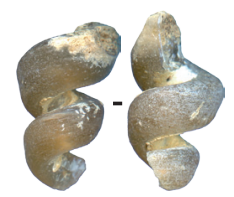

18

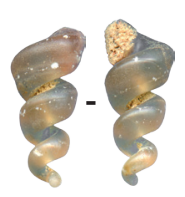

19

Fig. 8 - Parures d'Amiens-Renancourt 1 (Somme). 1 à 9 : fragments de rondelles en craie; 10 à 19 : moules internes silicifiés de turritelles (clichés 1 à $9:$ C. Peschaux et clichés 10 à $19:$ S. Lancelot INRAP).

Fig. 8 -Ornaments from Amiens-Renancourt 1 (Somme). 1 to 9: fragments of roundels in chalk; 10 to 19: internal silicified Turritella casts (photos 1 to 9: C. Peschaux and photos 10 to 19: S. Lancelot INRAP).

l'arrière ( $\mathrm{n}=3$ en comptant le nodule de silex) ou des hanches larges $(n=4)$. Une seule statuette, filiforme, ne correspond pas à ces canons (fig. 10d).

Les membres sont seulement esquissés, les bras notamment, sont courts et minces, plaqués contre la poitrine ou les flancs des statuettes $(n=6)$. Une statuette présente même une main gravée par quelques incisions (fig. 10b). Les membres inférieurs sont souvent arrêtés à hauteur de genoux, les cuisses séparées par une incision verticale. Il est plus difficile de se prononcer pour la tête, puisqu'elle n'est attestée que sur la première Vénus mise au jour (fig. 9) et un possible fragment (fig. 11d). L'absence répétée de cette partie sur toutes les autres interroge sur la volonté d'une fragmentation volontaire, ou d'une absence choisie lors du façonnage (segmentation de la figure). Ce choix de segmentation des représentations féminines ne pourra être éclairci qu'à la lueur des prochaines campagnes de fouilles et de nos travaux à venir sur le processus de fabrication des statuettes à Renancourt. 


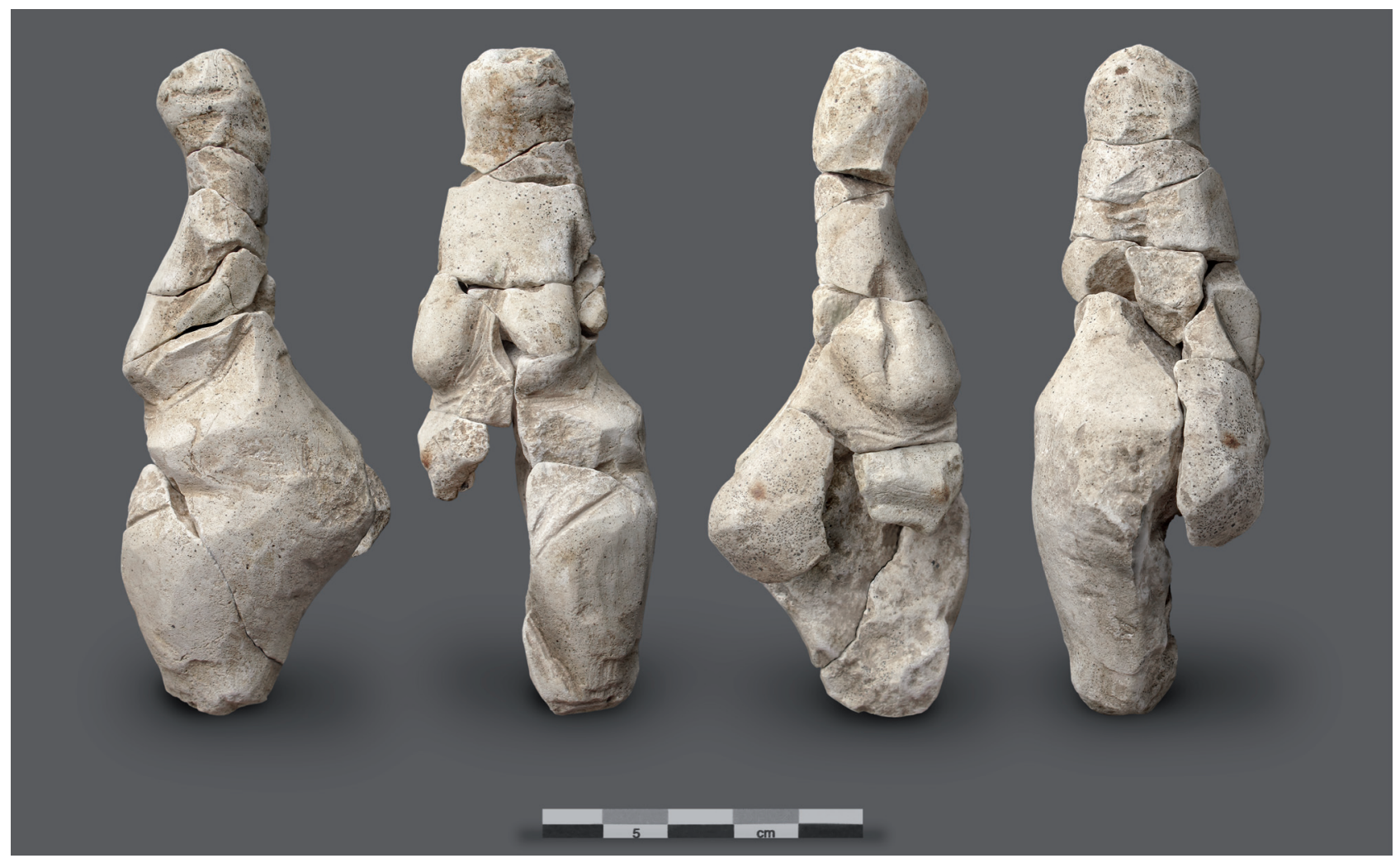

Fig. 9 - Amiens-Renancourt 1 (Somme), statuette $\mathrm{n}^{\circ} 861$ (photo S. Lancelot, INRAP).

Fig. 9 - Amiens-Renancourt 1 (Somme), statuette no. 861 (photo S. Lancelot, INRAP).

Ces caractéristiques stylistiques sont connues sur un vaste territoire, étendu du Sud-Ouest de la France jusqu'en Sibérie. Pourtant, les statuettes gravettiennes sont rares et souvent regroupées dans des aires géographiques relativement restreintes (Delporte, 1979; Otte, 1981 et 2013 ; Bourrillon, 2009; Simonet, 2012) : le NordOuest de l'Europe en était jusqu'ici dépourvu, à l'exception de deux fragments de statuettes sur le site gravettien rhénan de Mainz-Linsenberg (Hahn, 1969), et la sculpture en ivoire du Trou-Magrite à Pont-de-Lesse en Belgique (Otte, 1979) attribuée à l'Aurignacien (Lejeune, 1997).

Bien que l'on trouve une grande variété stylistique dans l'art mobilier gravettien, les sculptures découvertes à Amiens-Renancourt 1 appartiennent à cette tradition à plusieurs titres. La thématique de la représentation humaine d'abord est majoritairement féminine (au moins huit statuettes à Amiens-Renancourt 1) et la technique utilisée pour les éléments en craie est la ronde-bosse. D'ailleurs, si le calcaire n'est pas le matériau le plus fréquemment employé au Gravettien, son usage dans la sculpture d'objets en rondebosse se rencontre cependant sur les sites de Kostienki 1-I (Abramova, 1995; Dupuy, 2007) et Khotylevo 2 en Russie (Gavrilov, 2013), ainsi qu'à Willendorf en Autriche (Neugebauer-Maresch, 1999). L'état souvent fragmentaire de ces statuettes en calcaire est également un point de rapprochement. De même, la présence de possibles déchets de fabrication (nombreux fragments, amas et esquilles de calcaire qui ne semblent pas faire « naturellement » partie du substrat) fait écho à des vestiges de même type mises au jour sur les habitats de Kostienki 1, 4, 11 et 13, ou encore à Avdeevo en Russie (Abramova, 1995; Gvozdover, 1995).

Les conventions esthétiques d'Amiens-Renancourt 1 sont également identiques à celles des statuettes entières en pierre tendre ou en ivoire inventées sur tout le territoire gravettien. Ainsi, les détails anatomiques féminins sont mis en valeur, voire hypertrophiés, quand les membres sont esquissés, raccourcis ou absents, avec des figures acéphales. Les exemples sont nombreux comme notamment les célèbres Vénus de Lespugue en Haute-Garonne (Saint Périer, 1924), de Grimaldi en Italie (Bisson et White, 1996; White et Bisson, 1998), de Dolní Věstonice en Moravie (Delporte, 1979), de Gagarino en Russie (Abramova, 1995; Delporte, 1979), pour ne citer que les plus célèbres. Quelques statuettes sont toutefois plus «stylisées » avec des figures filiformes où seule la région médiane du corps est sexuée (ventre protubérant, rare indication de sexe) comme le sont par exemple les statuettes de Malta en Russie (Abramova, 1995), Tursac en Dordogne (Delporte, 1959) et la « fillette de Brassempouy » dans les Landes (White, 2006).

Enfin, les fragments découverts à Amiens montrent autant de stigmates de fabrication (raclage, rainurage, incision, polissage), et les techniques mises en œuvres devront être comparées à celles lisibles sur les statuettes en pierre tendre de Grimaldi (incisions profondes pour marquer la poitrine et les sillons inguinaux; White et Bisson, 1998), ou celles en marne calcaire de Kostienki (raclage, polissage, notamment sur les volumes : ventre, cuisses, fesses; Dupuy, 2007). 


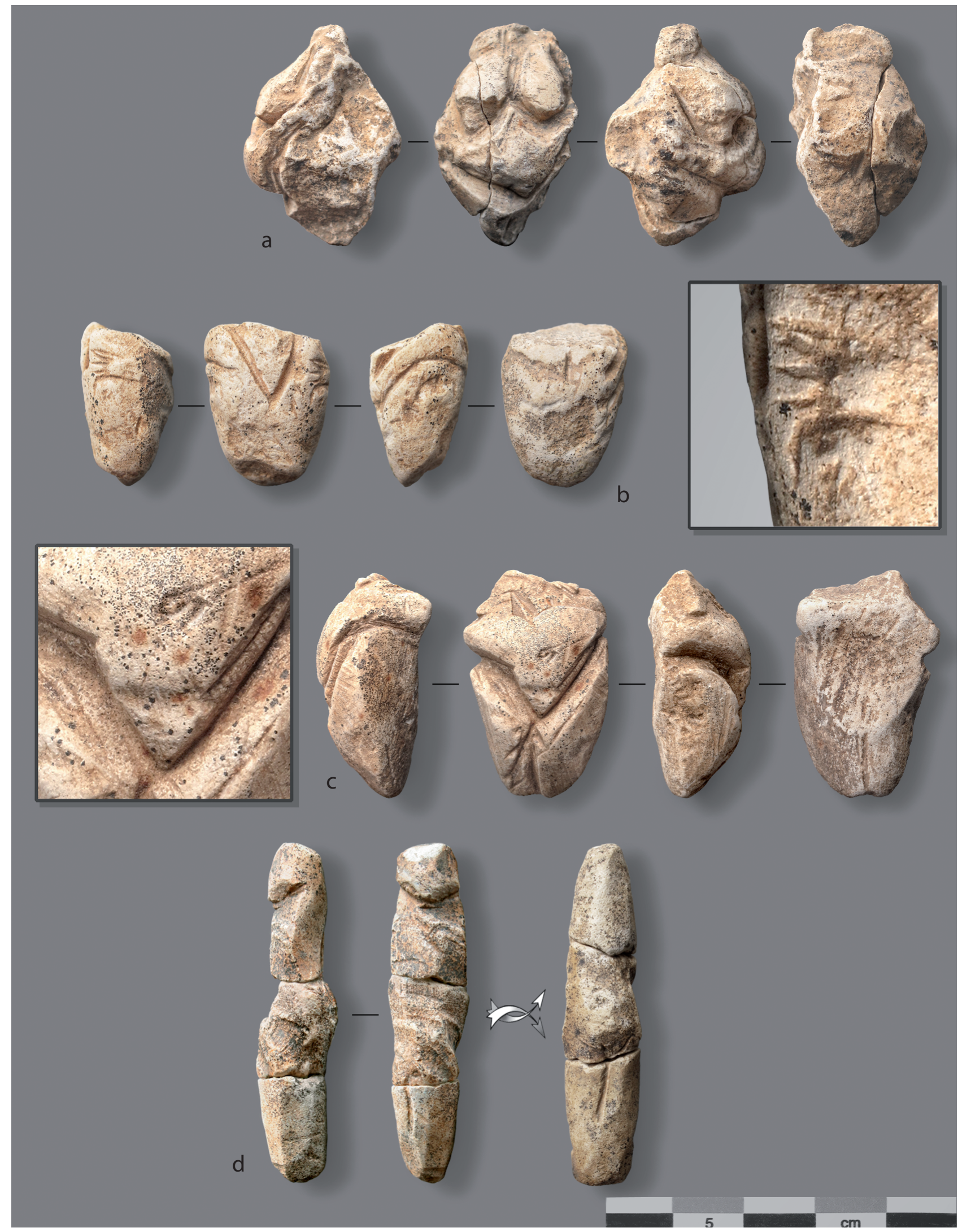

Fig. 10 - Amiens-Renancourt 1 (Somme), a : statuette $n^{\circ} 4393$; b : statuette ${ }^{\circ} 3562$; c : statuette ${ }^{\circ} 3982$; : statuette $n^{\circ} 3241$ (clichés S. Lancelot, INRAP).

Fig. 10 - Amiens-Renancourt 1 (Somme), a: statuette no. 4393; b: statuette no. 3562; c: statuette no. 3982; d: statuette no. 3241 (photos S. Lancelot, INRAP). 


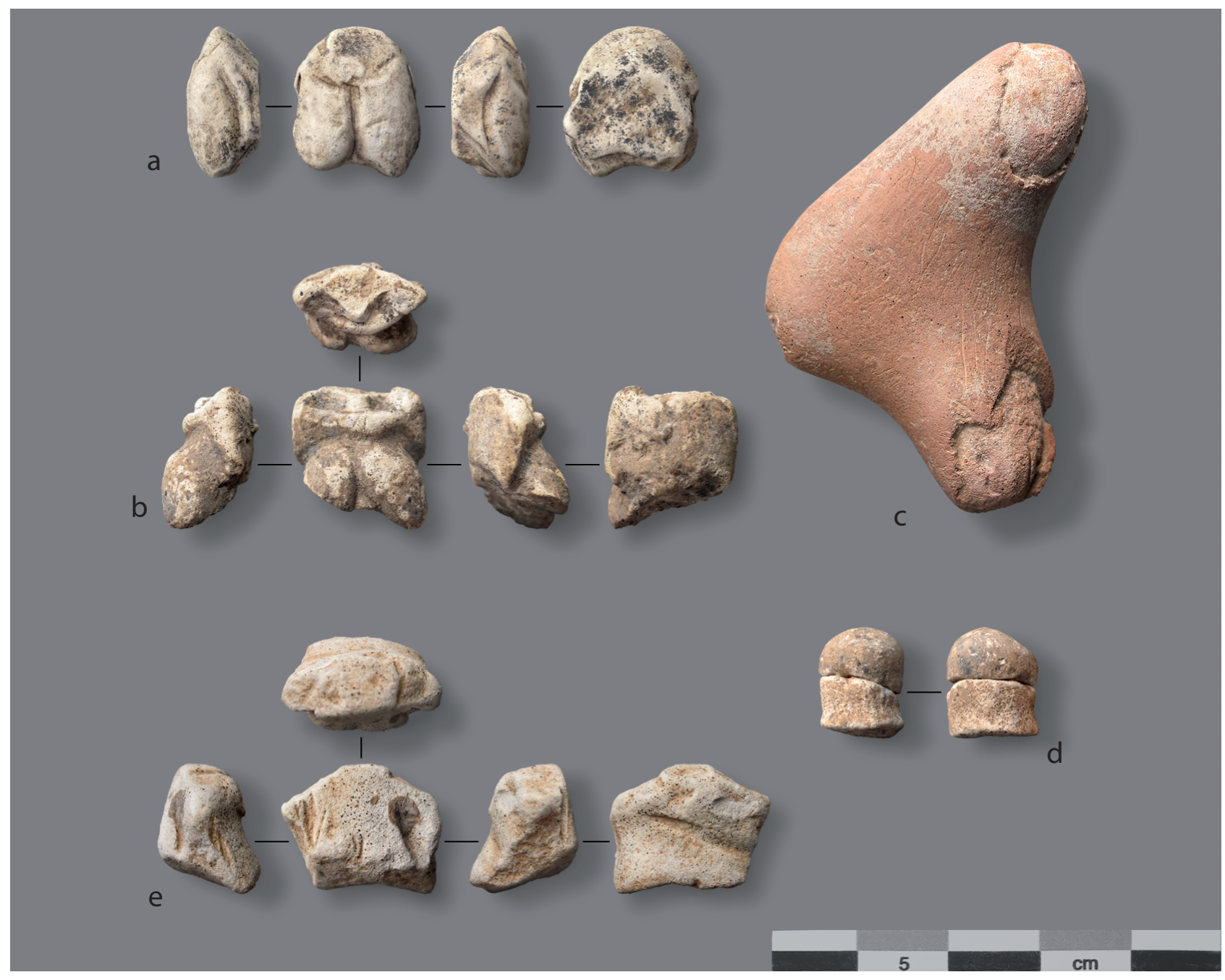

Fig. 11 - Amiens-Renancourt 1 (Somme), a : statuette $n^{\circ} 783$; b : statuette $n^{\circ} 6205$; c : nodule de silex $n^{\circ} 3488$; $d$ : ronde-bosse $n^{\circ} 4615$; e : statuette $n^{\circ} 3487$ (clichés S. Lancelot, INRAP).

Fig. 11 -Amiens-Renancourt 1 (Somme), a: statuette no. 783; b: statuette no. 6205; c: flint nodule no. 3488; d: sculpture in the round no. 4615; e: statuette no. 3487 (photos S. Lancelot, INRAP).

\section{PERSPECTIVES DE RECHERCHE SUR LES OBJETS EN CRAIE}

e nombre d'objets recueillis et leur état de conservation permettent d'envisager de stimulantes perspectives d'études. Un projet collectif de recherche (PCR) intitulé « Objets en craie d'Amiens-Renancourt 1 », a été mis place en 2016 sous la direction de C. Paris et E. Deneuve. Ce programme est complémentaire et interdépendant de l'opération de fouille programmée. Il engage une équipe d'une dizaine de chercheurs de différentes institutions (INRAP, ministère de la Culture, universités, CNRS) afin de procéder à des analyses physicochimiques et des tests expérimentaux sur la matière d'œuvre.

La localisation de la provenance de la craie et son acquisition par les Paléolithiques ainsi que les phénomènes de fracturation des objets en craie sont ainsi en cours d'étude. La fragmentation systématique des objets en craie fait notamment l'objet de plusieurs hypothèses non exclusives qu'il faudra tester. Il peut s'agir d'une fracturation accidentelle liée au façonnage ou d'une fracturation intentionnelle ultérieure. Cette dernière hypothèse est privilégiée depuis plusieurs années pour certains gisements russes ou ukrainiens (Iakovleva, 2013). Elle est néanmoins remise en cause sur le site de Kostienki I-1 par les travaux de D. Dupuy qui montre qu'il s'agirait plutôt d'une représentation intentionnelle de portions de corps (Dupuy, 2007). Par ailleurs, cette fracturation peut aussi résulter de phénomènes post-dépositionnels liés aux conditions climatiques rigoureuses périglaciaires (gélifraction).

Cette question des fractures est au cœur de la réflexion à mener pour comprendre la fonction et le statut du gisement d'Amiens-Renancourt 1. À l'inverse de la taille du silex, l'intentionnalité ou le caractère accidentel et postdépositionnel des fracturations de la craie est très difficile à déterminer. Cela est confirmé par la difficulté rencontrée lors des remontages d'amas de fragments de craie car aucune règle ne régit ces fractures à l'inverse du silex. 


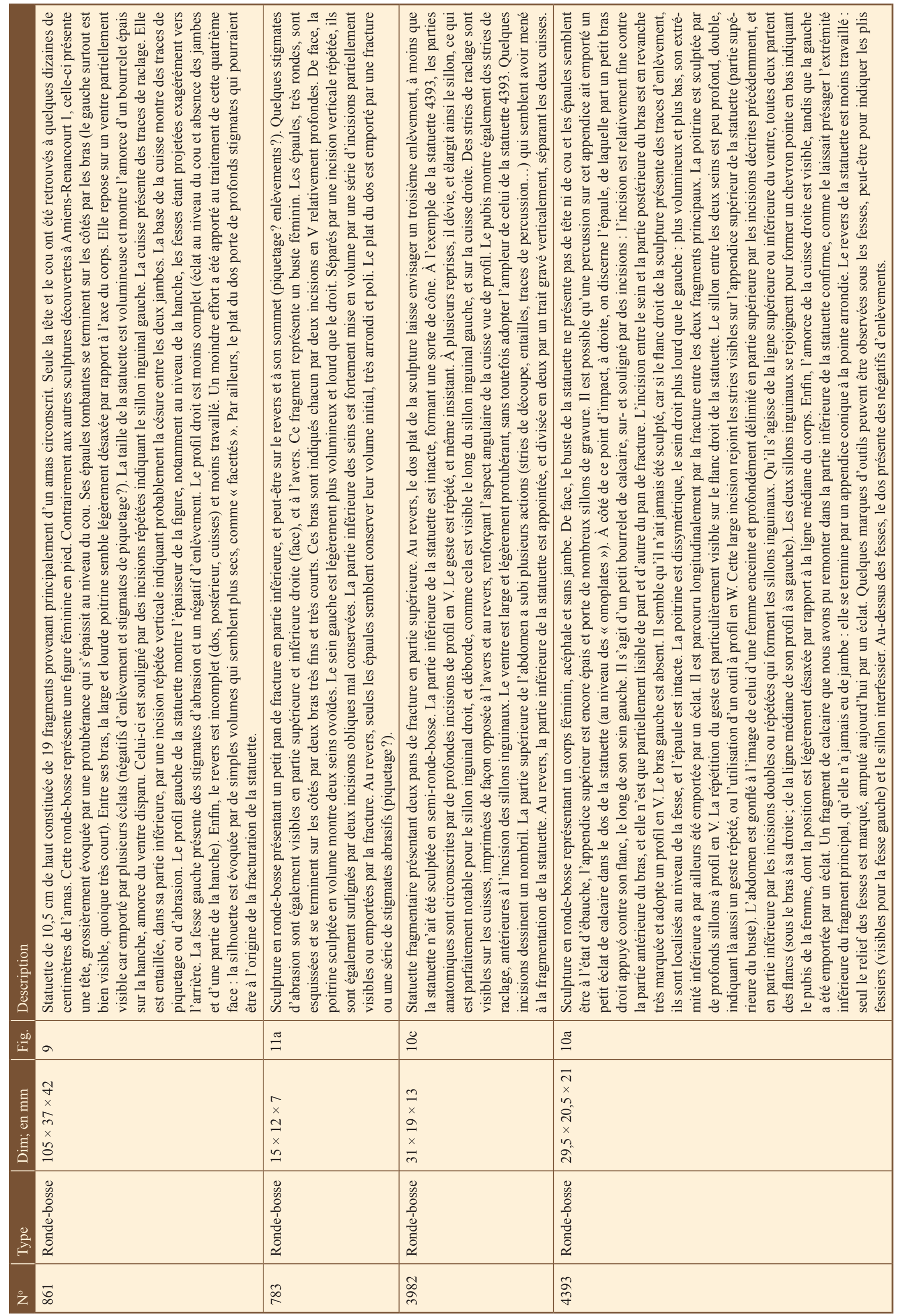




\begin{tabular}{|c|c|c|c|c|c|c|}
\hline & 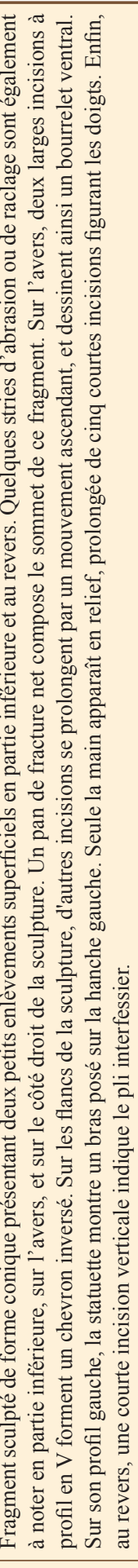 & 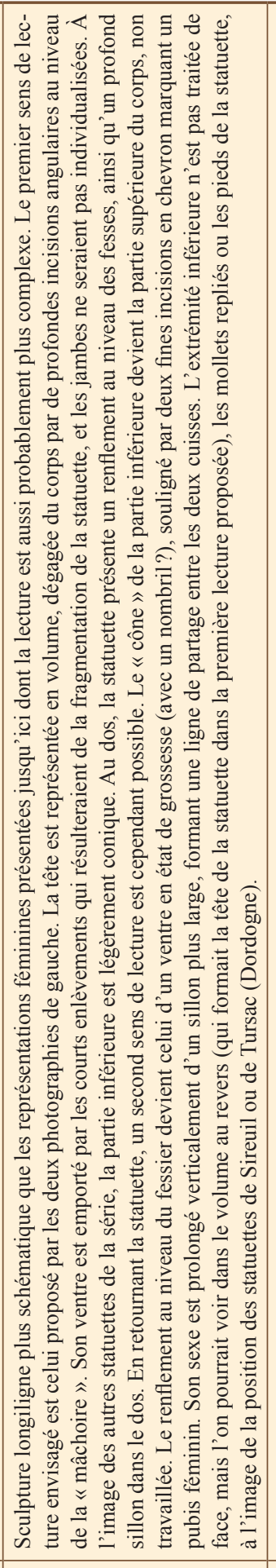 & 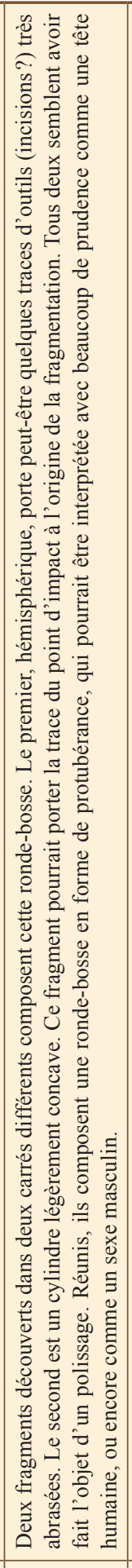 & 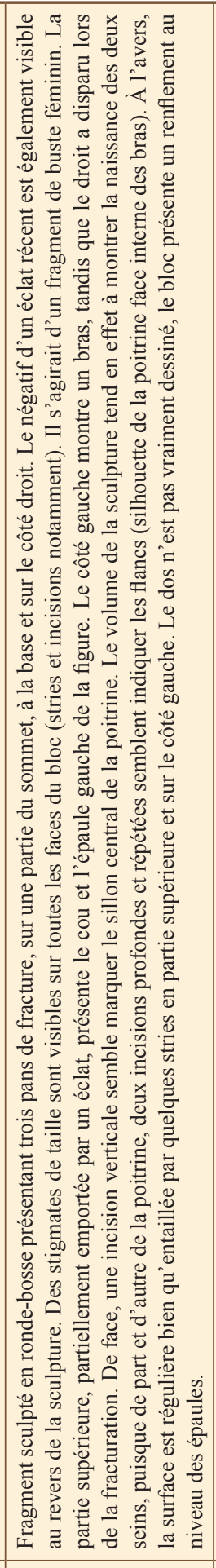 & 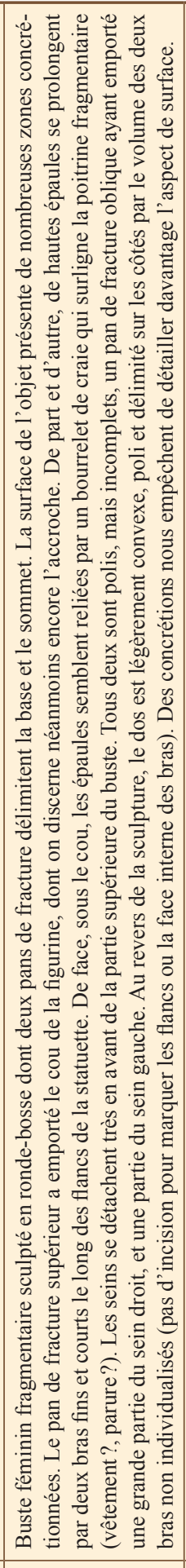 & 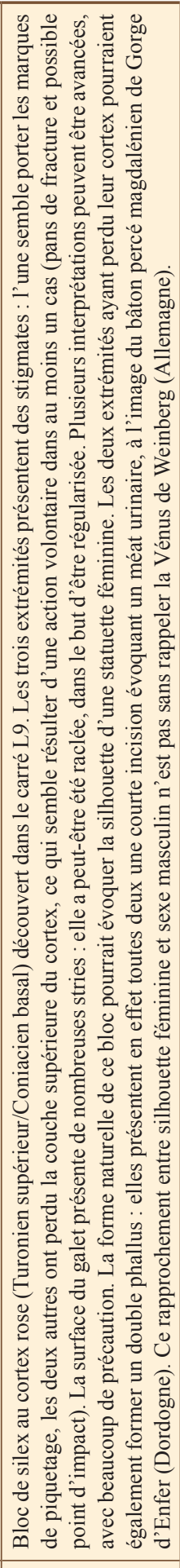 \\
\hline$\frac{00}{I I}$ & $\stackrel{0}{0}$ & $\stackrel{\square}{\Xi}$ & $\Xi$ & $\cong$ & $\fallingdotseq$ & $\stackrel{\cup}{=}$ \\
\hline & & $\begin{array}{l}n \\
a \\
x \\
x \\
x \\
x \\
o\end{array}$ & & $\begin{array}{l}\infty \\
x \\
x \\
m \\
\vec{x} \\
\underline{0} \\
\end{array}$ & $\begin{array}{l}r \\
x \\
n \\
m \\
x \\
x \\
\pm \\
\end{array}$ & $\begin{array}{l}\infty \\
\sim \\
x \\
\Sigma \\
x \\
x \\
i \\
n\end{array}$ \\
\hline & & 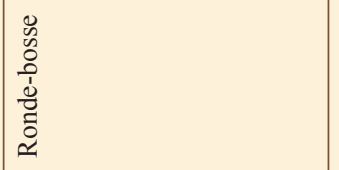 & 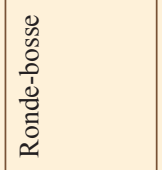 & $\begin{array}{l}0 \\
0 \\
0 \\
0 \\
\dot{d} \\
\tilde{\delta} \\
\tilde{0} \\
\simeq\end{array}$ & $\begin{array}{l}0 \\
0 \\
0 \\
0 \\
\dot{0} \\
\tilde{0} \\
\tilde{0}\end{array}$ & 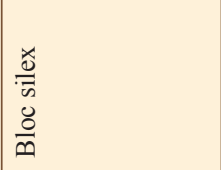 \\
\hline & & $\overline{\text { ते }}$ & $\frac{n}{6}$ & 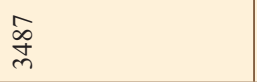 & స్రి & 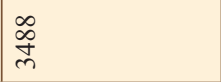 \\
\hline
\end{tabular}


La gélifraction de la craie sera donc expérimentée en laboratoire avec une reproduction de cycles de gel et de dégel similaire aux conditions climatiques contemporaines de l'occupation. La recherche de certains stigmates (impacts, points de percussion, flexions) et la localisation des fragments sur les plans de répartition des vestiges pourront également apporter des éléments quant à l'intentionnalité des fracturations.

Enfin, le nombre important de statuettes s'accompagne de plusieurs milliers de fragments de craie dont certains semblent être des déchets de fabrication (présence de traces anthropiques comme des stries par exemple). Leur nombre et leur bon état de conservation nous permettent d'envisager des études sur les techniques de fabrication mises en œuvre. Tous les objets en craie (statuettes et éléments de parure) présentent en effet de nombreuses traces de fabrication (raclage, polissage, rainurage...). Ces stigmates sont assez diversifiés pour référer aux différentes étapes du façonnage des sculptures. La diversité morphologique des objets sculptés, des fragments, et les différents stigmates qui y sont relevés, sont autant d'arguments en faveur d'un site de production d'objets d'art mobilier en craie, à l'image de ceux connus en Europe orientale. L'analyse de traces macro- et microscopiques permettra de les caractériser, et sans doute de déterminer les différents processus techniques mis en œuvre sur ce matériau. Cette analyse sera couplée à une étude tracéologique des outils en silex (J. Jacquier, en cours) ainsi qu'à une approche expérimentale qui permettra d'élaborer un corpus comparatif de stigmates. La méthodologie proposée est largement inspirée de la thèse de doctorat de D. Dupuy (2007) consacrée à l'étude des statuettes gravettiennes du site de Kostienki I-1. Elle aura pour but de déterminer le statut des pièces en craie recueillies sur le site en replaçant chaque élément en fonction de sa position dans la chaîne opératoire de fabrication.

\section{CONCLUSIONS}

$\mathrm{L}^{\mathrm{e}}$ e gisement d'Amiens-Renancourt 1 apporte une contribution importante à la connaissance du Paléolithique supérieur ancien du Nord de la France. La mise en évidence d'une occupation gravettienne incluse dans une séquence lœssique d'environ huit mètres de puissance, attribuable au Pléniglaciaire supérieur, comble une lacune dans l'histoire du peuplement précédant immédiatement le second maximum de froid du Weichselien. La richesse et le bon état de préservation de la documentation archéologique constituent un atout majeur dans le NordOuest européen au regard des trop rares gisements connus pour cette période. La présence de lœss calcaires a permis la bonne conservation des restes osseux, comme en témoigne l'existence d'éléments particulièrement fragiles (os long de fœtus). Parmi le spectre faunique le cheval apparaît comme l'espèce chassée dominante et certains éléments suggèrent une occupation du gisement durant la bonne saison entre le début du printemps et l'automne.
Les premières observations archéologiques montrent la présence d'une nappe de vestiges rapidement recouverte par la sédimentation loessique. Six datations ${ }^{14} \mathrm{C}$ placent l'occupation entre 22000 et 23000 BP, soit aux alentours de 27000 cal. BP. La découverte de nombreuses statuettes et d'éléments de parure confère un caractère exceptionnel au gisement d'Amiens-Renancourt 1. L'utilisation singulière de la craie est remarquable pour la fabrication de certaines parures et surtout de figurines humaines féminines. Le grand nombre d'objets découverts à ce jour souligne le statut particulier du gisement. Le travail de matériaux tendres comme certains calcaires ou la craie évoquent fortement les gisements de l'Europe centrale et orientale.

Les premières observations effectuées sur l'industrie lithique (Paris et al., 2013), caractérisée par une méthode de production de grandes lames obtenues au percuteur organique tendre, ainsi qu'une première mesure d'âge par la méthode du radiocarbone, nous avait incités à rapprocher l'industrie d'Amiens-Renancourt 1 du Gravettien final («Protomagdalénien » ou « Périgordien VII »; Bordes et Sonneville-Bordes, 1966). Certains éléments technotypologiques viennent cependant nuancer ce premier rapprochement avec les gisements " protomagdaléniens ». Contrairement au stade ultime du Gravettien, les fragments de pièces à dos d'Amiens-Renancourt 1 ne présentent pas de troncature. La présence de quelques microgravettes et de pointes de la Gravette nous éloigne également du corpus des armatures des sites attribués au Gravettien final où ces deux éléments de projectiles sont généralement très rares ou absents. De plus, les nouvelles datations d'Amiens-Renancourt 1 sont légèrement plus anciennes que celles du «Protomagdalénien » (voir notamment Guillermin, 2011). Les rapprochements actuels s'orientent davantage vers les sites rhénans à l'exemple de Mainz-Linsenberg (Hahn, 1969) ou Sprendlingen (Bosinski et al., 1985) qui montrent de fortes similarités dans l'industrie lithique, notamment au niveau des armatures et par la présence sur le site de Mainz de deux fragments de statuettes féminines. Ces deux gisements, comme un certains nombre de sites du Sud-Ouest de la France, ont été récemment rassemblés dans un faciès récent, ou récent-final du Gravettien (Klaric et al., 2009; Klaric, 2013). L'industrie du gisement d'AmiensRenancourt 1 pourrait donc être rapportée à l'un de ces faciès. La seule datation ${ }^{14} \mathrm{C}$ disponible pour le gisement de Mainz-Linsenberg, bien que considérée comme probablement trop récente par T. Terberger (2013), confirme cette attribution et place l'occupation archéologique de ce gisement en parallèle avec celle d'Amiens-Renancourt 1. Les études ultérieures permettront de préciser les relations entre l'industrie d'Amiens-Renancourt 1 et les différents faciès qui caractérisent la fin du Gravettien.

Les manifestations d'ordre esthétique recueillies à ce jour contribuent également à nuancer l'attribution chronoculturelle initialement proposée (Paris et al., 2013). Les éléments de parure s'écartent effectivement de ce que l'on connaît pour le Gravettien final. Les deux types recueillis à Amiens-Renancourt 1 se distinguent de 
la parure du «Protomagdalénien » caractérisée par des perles rectangulaires à perforation et amincissements centraux (Allard et al., 1997). Les éléments de comparaison pour les rondelles en craie se trouvent davantage dans l'Est de l'Europe et en particulier dans la sépulture de Brno II, en République tchèque, qui a livré une dizaine de rondelles en os, pierre, dent et ivoire. Cette sépulture est par ailleurs datée d'environ 23000 BP non cal. (Oliva, 2000). En ce qui concerne les moules internes silicifiés de Turritelles, le seul site connu actuellement pour en avoir livré un grand nombre (180 exemplaires) est celui de la troisième grotte de Goyet en Belgique (Otte, 1979; Dewez, 1987; Moreau, 2003). Le niveau dans lequel ces parures ont été retrouvées est classiquement attribué au
Magdalénien mais une attribution au Gravettien n'est peut-être pas à exclure compte tenu de la grande ancienneté des fouilles réalisées dans ce gisement. Quant aux statuettes féminines, il semble qu'elles soient généralement absentes des sites du Gravettien final. Seul un objet dans la couche 2 de l'abri Pataud, d'abord interprété comme une statuette animale, a été reconsidéré comme une possible statuette féminine avec beaucoup de précaution (White, 2002; Chiotti et al., 2009). Les rapprochements les plus pertinents s'établissent donc avec le Gravettien récent ou récent-final et notamment avec le gisement de MainzLinsenberg en Allemagne où deux fragments de statuettes féminines ont été découverts (Hahn, 1969).

\section{RÉFÉRENCES BIBLIOGRAPHIQUES}

Abramova Z. A. (1995) - L'art paléolithique d'Europe orientale et de Sibérie, Grenoble, Jérôme Million (L'Homme des origines), $367 \mathrm{p}$.

Allard M., Drieux M., Jarry M., Pomies M.-P., Rodière J. (1997) - Perles en bois de renne du niveau 18 des Peyrugues, à Orniac (Lot) : hypothèse sur l'origine du Protomagdalénien, Paléo, 9, p. 355-369.

Antoine P. (1991) - Nouvelles données sur la stratigraphie du Pléistocène supérieur de la France septentrionale, d'après les sondages effectués sur le tracé du TGV Nord, in A. Truffeau (dir.), Paléolithique et Mésolithique du Nord de la France; nouvelles recherches, 2, Lille, CERP (Publications du Centre d'études et de recherches préhistoriques de l'université des sciences et technologies de Lille, 3), p. 9-20.

Antoine P., Rousseau D.-D., Moine O., Kunesch S., Hatte C., LANG A., Zoller L. (2009) - Evidence of Rapid and Cyclic Eolian Deposition during the Last Glacial in European Loess Series (Loess Events): The High-resolution Records from Nussloch (Germany), Quaternary Science Reviews, 28, p. 2955-2973.

Antoine P., Goval E., Jamet G., Coutard S., Moine O., Hérisson D., Auguste P., Guérin G., Lagroix F., Schmidt E. (2014) - Les séquences loessiques Pléistocène supérieur d'Havrincourt (Pas-de-Calais, France) : stratigraphie, paléoenvironnements, géochronologie et occupations paléolithiques, Quaternaire, 25, 4, p. 321-368.

Antoine P., Coutard S., Guérin G., Deschodt L., Goval É., Locht J.-L., PARIS C. (2016) - Upper Pleistocene Loesspalaeosols Records from Northern France in the European Context: Environmental Background and Dating of the Middle Palaeolithic, Quaternary international, 411, p. 4-34.

Bisson M., White R. (1996) - L'imagerie féminine du Paléolithique : étude des figurines de Grimaldi, Culture, 26, 2, p. 5-60.

Bordes F., Sonneville-Bordes D. de (1966) - Protomagdalénien ou Périgordien VII?, L'Anthropologie, 70, 1-2, p. 113-122.

Bosinski G., Bosinski H., Brunnacker K., Cziesla E., Lanser K.-P., Neuffer O., Preuss J., Spoerer H., Tillmanns W., Urban B. (1985) - Sprendlingen. Ein Fundplatz des Mittleren Jungpaläolithikums in Rheinhessen, Jahrbuch des Römisch-Germanischen Zentralmuseums, 32, p. 5-91.
BOURRILLON R. (2009) - Les représentations humaines sexuées dans l'art du Paléolithique supérieur européen : diversité, reminiscences et permanences, thèse de doctorat, université Toulouse 2 - Le Mirail, 552 p.

Chiotti L., Nespoulet R., Henry-Gambier D., Morala A., Vercoutère C. avec la collaboration de S. Agsous, A. Lenoble, L. Marquer, D. Grimaud-Hervé (2009) - Statut des objets « extra-ordinaires » du Gravettien final de l'abri Pataud (Les Eyzies-de-Tayac, Dordogne) : objets abandonnés dans l'habitat ou dépôt intentionnel? in $\mathrm{S}$. Bonnardin, C. Hamon, M. Lauwers et B. Quilliec (dir.), Du matériel au spirituel. Réalités archéologiques et historiques des dépôts de la Préhistoire à nos jours, actes des XXIX ${ }^{\text {es }}$ Rencontres internationales d'archéologie et d'histoire (Antibes, 15-18 octobre 2008), Juan-les-Pins, APDCA, p. 29-46.

Commont V. (1913) - Les hommes contemporains du renne dans la vallée de la Somme, Amiens, Société des antiquaires de Picardie (Mémoire, 37), p. 207-646.

Delporte H. (1959) - Une nouvelle statuette paléolithique : la Vénus de Tursac, L'Anthropologie, 63, 3-4, p. 233-247.

Delporte H. (1979) - L'image de la femme dans l'art préhistorique, Paris, Picard, $320 \mathrm{p}$.

Dewez M. (1987) - Le Paléolithique supérieur récent dans les grottes de Belgique, Louvain-la-Neuve, universtié catholique de Louvain (Publication d'histoire de l'art et d'archéologie de l'université catholique de Louvain, 57), 466 p.

Dupuy D. (2007) - Fragments d'images, images de fragments. La statuaire gravettienne, du geste au symbole, thèse de doctorat, université Provence-Aix-Marseille I, Aixen-Provence, $321 \mathrm{p}$.

Fagnart J.-P. (1980) - Le Paléolithique supérieur dans le bassin de la Somme d'après les recherches de V. Commont, Cahiers archéologiques de Picardie, 7, p. 19-32.

FAgnART J.-P. (1988) - Les industries lithiques du Paléolithique supérieur dans le Nord de la France, Amies, direction des Antiquités de Picardie (Revue archéologique de Picardie, numéro spécial), $153 \mathrm{p}$.

Fagnart J.-P., Coudret P., Antoine P., avec la collaboration de Vallin L., Sellier N., Masson B. (2013) - Le Paléolithique supérieur ancien dans le Nord de la France, in P. Bodu, L. Chehmana, L. Klaric, L. Mevel, S. Soriano 
et N. Teyssandier (dir.), Le Paléolithique supérieur ancien de l'Europe du Nord-Ouest. Réflexions et synthèses à partir d'un projet collectif de recherche sur le Centre et le Sud du Bassin parisien, actes du colloque de Sens (15-18 avril 2009), Paris, Société préhistorique française (Mémoire, 56), p. $197-214$.

FAgnart J.-P., Coudret P. (1997) - Amiens-Renancourt, rue Haute-des-Champs, Bilan scientifique de la région Picardie, Amiens, service régional de l'Archéologie de Picardie, p. 86.

Font C., Coutard S., Paris C., Hérisson D., Goval É., Locht J.-L. (2016) - Formations superficielles et occupations paléolithiques en France septentrionale : développement et premières applications d'un système d'information géographique (SIG), Revue archéologique de Picardie, 1, 2, p. 41-68.

GavriLov K. N. (2013) - Nouvelles figures féminines du site de Khotylevo 2, in J. Clottes (dir.), L'art pléistocène dans le monde, actes du congrès IFRAO, symposium 8 (Tarasconsur-Ariège, septembre 2010), Tarascon-sur-Ariège, Société préhistorique Ariège-Pyrénées, p. 1291-1297.

Goval É., Herisson D. (2012) - Découverte inédite de trois occupations du Pléniglaciaire moyen du Weichselien à Havrincourt « les Bosquets » (Pas-de-Calais, France), Bulletin de la Société préhistorique française, 109, 2, p. 342345.

Guillermin P. (2011) - La fin du Gravettien dans le SudOuest de la France : à la recherche de l'identité protomagdalénienne, in N. Goutas, L. Klaric, D. Pesesse et P. Guillermin (dir.), À la recherche des identités gravettiennes : actualités, questionnements et perspectives, actes de la table ronde sur le Gravettien en France et dans les pays limitrophes (Aix-en-Provence, 6-8 octobre 2008), Paris, Société préhistorique française (Mémoire, 52), p. 129-144.

Gvozdover M.-G. (1995) - Art of the Mammoth Hunters: the Finds of Avdeevo, Paris, CNRS, 223 p.

HAHN J. (1969) - Gravettien-Freilandstationen im Rheinland: Mainz-Linsenberg, Koblenz-Metternich und Rhens, Bonner Jahrbücher, 169, p. 44-87.

KLARIC L. (2013) - Faciès lithiques et chronologie du Gravettien du Sud du Bassin parisien et de sa marge sud-occidentale, in P. Bodu, L. Chehmana, L. Klaric, L. Mevel, S. Soriano et N. Teyssandier (dir.), Le Paléolithique supérieur ancien de l'Europe du Nord-Ouest. Réflexions et synthèses à partir d'un projet collectif de recherche sur le Centre et le Sud $d u$ Bassin parisien, actes du colloque (Sens, 15-18 avril 2009), Paris, Société préhistorique française (Mémoire, 56), p. 61-87.

Klaric L., Guillermin P., Aubry T. (2009) - Des armatures variées et des modes de productions variables : réflexions à partir de quelques exemples issus du Gravettien d'Europe occidentale (France, Portugal, Allemagne), Gallia Préhistoire, 51, p. 113-154.

IAKOvLEVA L. (2013) - L'art mobilier du Gravettien. in M. Otte (dir.), Les Gravettiens, Paris, Errance (Civilisations et Cultures), p. 237-270.

Lejeune M. (1997) - L'art mobilier du Trou Magrite (Pontà-Lesse, Belgique) : Aurignacien ou Gravettien?, L'Anthropologie, 101, 1, p. 238-247.
Moreau L. (2003) - Les éléments de parure au Paléolithique supérieur en Belgique, L'Anthropologie, 107, p. 603-614.

Neugebauer-Maresch C. (1999) - Le Paléolithique en Autriche, Grenoble, Jérôme Million (Préhistoire d'Europe, 8), $202 \mathrm{p}$.

Oliva M. (2000) - The Brno II Upper Palaeolithic Burial, in W. Roebroeks, M. Mussi, J. Svoboda et K. Fennema (éd.), Hunters of the Golden Age. The Mid Upper Palaeolithic of Eurasia 30,000-20,000 BP, Leyde, University of Leiden (Analecta Praehistorica Leidensia, 31), p. 143-153.

Отте M. (1979) - Le Paléolithique supérieur ancien en Belgique, Bruxelles, Musées royaux d'art et d'histoire, $684 \mathrm{p}$.

Отте M. (1981) - Le Gravettien en Europe centrale, Bruges, De Tempel (Dissertationes Archaeologicae Gandenses, 20), 2 vol., 505 p.

Отте M., dir. (2013) - Les Gravettiens, Paris - Arles, Errance (Civilisations et Cultures), $351 \mathrm{p}$.

Paris C., Fagnart J.-P., Coudret P. (2013) - Du Gravettien final dans le Nord de la France? Nouvelles données à Amiens-Renancourt, Bulletin de la Société préhistorique française, 110, 1, p. 123-126.

Paris C., dir. (2015) - Renancourt 2 : un site du Paléolithique ancien dans le Nord de la France, rapport final d'opération, INRAP Nord-Picardie, service régional de l'Archéologie de Picardie, Amiens, 194 p.

Paris C., Antoine P., Auguste P., Claud E., Coutard S., Coudret P., Deneuve E., Goutas N., Fagnart J.-P., Font C., Lacarrière J., Moine O., Peschaux C. (à paraitre) - Les gisements gravettiens d'Amiens-Renancourt : premières données palethnologiques, in C. Montoya, J.-P. Fagnart et J.-L. Locht, Préhistoire de l'Europe du NordOuest. Mobilités, climats et identités culturelles, actes du XXVIII ${ }^{\mathrm{e}}$ Congrès préhistorique de France, session 2 (Amiens, 30 mai - 4 juin 2016), Paris, Société préhistorique française.

Pelegrin J. (2000) - Les techniques de débitage laminaires au Tardiglaciaire : critères de diagnose et quelques réflexions; in B. Valentin, P. Bodu et M. Christensen (dir.), L'Europe centrale et septentrionale au Tardiglaciaire, actes de la table ronde internationale (Nemours, 14-16 mai 1997), Nempurs, APRAIF, p. 73-86.

Rasmussen S. O., Bigler M., Blockley S. P., Blunier T., Buchardt S. L., Clausen H. B., Cvijanovic I., DahlJensen D., Johnsen S. J., Fischer H., Gkinis V., GuiLLevic M., Hoek W. Z., Lowe J. J., Pedro J. B., Popp T., Seierstad I. K., Steffensen J. P., Svensson A. M., VaLLelonga P., Vinther B. M., Walker M. J. C., Wheatley J. J., WinstruP M. (2014) - A Stratigraphic Framework for Abrupt Climatic Changes during the Last Glacial Period Based on Three Synchronized Greenland Ice-core Records: Refining and Extending the INTIMATE Event Stratigraphy, Quaternary Science Reviews, 106, p. 14-24.

Reimer P. J., Bard E., Bayliss A., Beck J. W., Blackwell P. G., Bronk Ramsey C., Buck C. E., Cheng H., Edwards R. L., Friedrich M., Grootes P. M., Guilderson T. P., Haflidason H., Hajdas I., Hatté C., Heaton T. J., Hoffmann D. L., Hogg A. G., Hughen K. A., Kaiser K. F., Kromer B., Manning S. W., Niu M., Reimer R. W., Richards D. A., Scott E. M., Southon 
J. R., Staff R. A., Turney C. S. M., Van Der Plicht J. (2013) - Intcal13 and Marine13 Radiocarbon Age Calibration Curves 0-50,000 Years Cal. BP, Radiocarbon, 55, 4, p. $1869-1887$.

SAINT-PÉRIER R. de (1924) - La statuette féminine de Lespugue (Haute-Garonne), Bulletin de la Société préhistorique française, 21, 3, p. 81-84.

Simonet A. (2012) - Brassempouy (Landes, France) ou la matrice gravettienne de l'Europe, Liège, université de Liège (ERAUL, 133), $141 \mathrm{p}$.

Terberger T. (2013) - Le Dernier Maximum Glaciaire entre le Rhin et le Danube, un réexamen critique, in P. Bodu, L. Chehmana, L. Klaric, L. Mevel, S. Soriano et N. Teyssandier (dir.), Le Paléolithique supérieur ancien de l'Europe du Nord-Ouest. Réflexions et synthèses à partir d'un projet collectif de recherche sur le Centre et le Sud du Bassin parisien, actes du colloque (Sens, 15-18 avril 2009), Paris, Société préhistorique française (Mémoire, 56), p. 415-443.

White R., Bisson M. (1998) - Imagerie féminine du Paléolithique : l'apport des nouvelles statuettes de Grimaldi, Gallia Préhistoire, 40, p. 95-132.

White R. (2002) - Une nouvelle statuette phallo-féminine paléolithique : « La Vénus des Milandes » (commune de Castelnaud-la-Chapelle, Dordogne), Paléo, 14, p. 177-198.

White R. (2006) - The Women of Brassempouy: A Century of Research and Interpretation, Journal of Archaeological Method and Theory, 13, 4, p. 251-304.

\section{Clément PARIS}

INRAP Hauts-de-France, centre archéologique de Passel, parc d'activités, avenue du Parc, 60400 Passel et UMR 7041 ArScAn, équipe Ethnologie préhistorique clement.paris@inrap.fr

Émeline Deneuve conservatrice du Patrimoine, MCC, INRAP Hauts-de-France, 32, avenue de l'Étoile-du-Sud, 80440 Glisy et UMR $7194 \mathrm{du}$ MNHN. emeline.deneuve@inrap.fr

\section{Jean-Pierre FAGNART}

conseil départemental de la Somme, direction de la Culture et des Patrimoines, 54, rue Saint-Fuscien, BP 32615 , 80026 Amiens cedex jp.fagnart@somme.fr
Paule Coudret

18, rue Dufour, 80000 Amiens p.coudret@wanadoo.fr

Pierre Antoine Laboratoire de géographie physique, environnements quaternaires et actuels, UMR 8591, CNRS, université Paris I, UPEC, 1, place A. Briand, 92195 Meudon Pierre.Antoine@cnrs-bellevue.fr

Jessica LACARRIÈRE Chercheur associé, UMR 5608 TRACES, équipe smp $3 \mathrm{c}$, Maison de la recherche, université Toulouse Jean-Jaurès, 5, allées Antonio-Machado, 31058 Toulouse cedex 9 jessic.laca@gmail.com

Caroline Peschaux UMR 7041 ArScAn, université Paris-Ouest Nanterre-La Défense, CNRS, équipe Ethnologie préhistorique, Maison archéologie et ethnologie, 21, allée de l'université, 92023 Nanterre cedex et université Paris 1 - Panthéon-Sorbonne caroline.peschaux@malix.univ-paris1.fr

Sylvie Coutard

INRAP Hauts-de-France, 32, avenue de l'Étoile-du-Sud, 80440 Glisy et UMR 8591, CNRS, Laboratoire de géographie physique, environnements quaternaires et actuels sylvie.coutard@inrap.fr

Olivier MoIne UMR 8591, CNRS, Laboratoire de géographie physique, environnements quaternaires et actuels, université Paris I, UPEC,

1, place Aristide-Briand, 92195 Meudon olivier.moine@cnrs-bellevue.fr

Gilles GUÉRIN

GEOTRAC, LSCE, bât. 12, avenue de la Terrasse, 91198 Gif-sur-Yvette gilles.Guerin@1sce.ipsl.fr 